\title{
Signal-background interference for digluon resonances at the Large Hadron Collider
}

\author{
Prudhvi N. Bhattiprolu $\odot$ and Stephen P. Martin $\odot$ \\ Department of Physics, Northern Illinois University, DeKalb, Illinois 60115, USA
}

(Received 24 April 2020; accepted 2 July 2020; published 16 July 2020)

\begin{abstract}
We study the interference between the amplitudes for $g g \rightarrow X \rightarrow g g$, where $X$ is a new heavy digluon resonance, and the QCD background $g g \rightarrow g g$, at the Large Hadron Collider. The interference produces a large low-mass tail and a deficit of events above the resonance mass, compared to the naive pure-resonance peak. For a variety of different resonance quantum numbers and masses, we evaluate the signal-background interference contribution at leading order, including showering, hadronization, and detector effects. The resulting new physics dijet mass distribution may have a shape that appears, after QCD background fitting and subtraction, to resemble an enhanced peak, a shelf, a peak/dip, or even a pure dip. We argue that the true limits on new digluon resonances are likely to differ significantly from the limits obtained when interference is neglected, especially if the branching ratio to $g g$ is less than 1 .
\end{abstract}

DOI: 10.1103/PhysRevD.102.015016

\section{INTRODUCTION}

Understanding the dijet invariant-mass spectrum provides an essential way to discover or set limits on certain types of new physics beyond the Standard Model, as new particles could decay primarily to $g g, q \bar{q}, q q$, or $q g$. The most recent Large Hadron Collider (LHC) searches in the dijet channel can be found in Refs. [1,2] (CMS) and Refs. [3,4] (ATLAS), based in part on strategies developed in earlier searches in Refs. [5-11]. Some models of phenomenological interest that yield dijet signals include the following. Chiral color [12-18], flavor-universal coloron [19-30], and certain supersymmetric models [31] predict the existence of massive color-octet gauge bosons, because of the embedding of QCD within a broken symmetry of the $S U(3) \times S U(3)$ gauge group beyond the $\mathrm{TeV}$ scale. These massive gauge bosons are known as axigluons (axial vectors) or colorons (vectors), which typically decay to a $q \bar{q}$ pair, or topgluons [32-36], which can preferentially couple to $t$ quarks and appear in topcolor and similar models of dynamical electroweak symmetry breaking. Some $E_{6}$ grand unified theories predict diquarks [37] which decay to $q q$. Models with new electroweak gauge bosons $W^{\prime}$ and $Z^{\prime}[38,39]$, on top of the Standard Model, could also decay to a $q \bar{q}$ pair. Excited or composite quarks $[40,41]$ could decay to $q g$ or $\bar{q} g$. String Regge resonances $[42,43]$ of the quark and the gluon could decay

Published by the American Physical Society under the terms of the Creative Commons Attribution 4.0 International license. Further distribution of this work must maintain attribution to the author(s) and the published article's title, journal citation, and DOI. Funded by SCOAP ${ }^{3}$. to a quark and a gluon. Scalar color octets, which occur in models of technicolor [44] and universal extra dimensions $[45,46]$, can be considered as digluon resonances. The Randall-Sundrum (RS) model [47], which provides a possible solution to the Planck-scale hierarchy problem by adding extra dimensions, predicts RS gravitons which decay to gluon pairs or quark pairs. The Kaluza-Klein (KK) states interact with the Standard Model fields, and thus the KK gravitons could contribute to the dijet spectrum [48]. Also, models with dark matter mediators [49-52] that also couple to quarks predict dijet signatures. A general classification and study of dijet resonances for the LHC has been given in Ref. [53].

One of the biggest challenges in limiting or discovering a new dijet resonance is dealing with the huge QCD background, which is imperfectly known but should be a smooth function of the invariant mass in the range appropriate for new physics searches. In order to effectively deal with the background, for lower masses CMS uses the data scouting [54] technique to reconstruct or save only the crucial information to do analyses, thus allowing them to record more events. In the CMS and ATLAS searches that set limits on dijet resonances, the interference between the resonant amplitude and the QCD background was not considered, which could have a significant impact on the experimental limits. As we will see below, the interference effect means that dijet resonances need not necessarily produce a peak in the dijet mass distributions in the vicinity of their mass, especially once the QCD background fitting and subtraction are implemented. The effects of interference are likely to be most pronounced in the digluon channel, where the QCD background amplitudes are large compared to the new-physics amplitudes. A preliminary 
study of this kind of interference effect for digluon resonances, done only at parton level with smearing and only for the case of a spin- 0 color singlet with mass near $750 \mathrm{GeV}$ (motivated in large part by an infamous possible diphoton signal that turned out to be a fluctuation), was performed in Ref. [55].

A similar but much smaller effect on the diphoton line shape for the Higgs boson due to interference with the quarkloop-induced Standard Model amplitudes $g g \rightarrow \gamma \gamma$ has been studied in Refs. [56-65]. As noted in Ref. [58], there is a shift in the diphoton mass peak which can eventually be observable, and can be used [61] to bound the Higgs boson width. Another important case of interference involving Standard Model Higgs-boson-mediated amplitudes and the continuum contributions occur for the processes $g g \rightarrow Z Z$ and $g g \rightarrow$ $W^{+} W^{-}$, which have been studied in Refs. [66-87]. In particular, as noted in Ref. [74], enhanced contributions occur for invariant masses far off the Higgs mass shell, despite its narrow width, and are reduced by the interference. As shown in Refs. [77,78] (see Refs. [79-87] for further important developments), this effect can be (and has been [88-93]) used to bound the Higgs width from studying $V V$ events in the invariant-mass region far above the Higgs mass at the LHC. Other aspects of resonance-continuum interferences as a probe of new physics at the LHC, with approaches similar or complementary to the present paper, have been given in Refs. [94-100]. Also, Ref. [101] discussed the importance of noninterference off-shell effects in spin-1 digluon resonances at hadron colliders.

In this paper, we consider digluon resonances of various spin and color quantum numbers, whose existence need not necessarily be justified by any particular model. We study the importance of the interference between the digluon resonant signal $g g \rightarrow X \rightarrow g g$ and the QCD background $g g \rightarrow g g$ when setting limits on the digluon resonances, where $X$ couples to gluon pairs by nonrenormalizable operators, subject to QCD gauge invariance. Here and from now on, $X$ refers to any such digluon resonance.

At leading order (LO) the interference terms change the naive Breit-Wigner resonance peak, at dijet invariant mass close to the resonance mass $\left(m_{j j} \approx M_{X}\right)$, to a peak just below and a dip just above the resonance mass. [As a caution, we note that a next-to-leading-order (NLO) calculation with virtual one-loop and real emission of an extra jet would provide a more realistic estimate; NLO effects can be quite significant for the interference in the analogous case of diphoton Higgs signal/background interference [59-62], especially when there is an additional jet with high $p_{T}$. ] The magnitudes of these interference effects are dependent on the spin and the color structures of the digluon resonances. The interference effects are studied for scalar and pseudoscalar resonances in both singlet and octet color representations, spin-1 color-octets, and color-singlet massive gravitons. Although the Landau-Yang theorem forbids the decay of a massive spin-1 particle into two on-shell photons, it does not forbid the decay of an oddparity massive color-octet spin-1 particle into a pair of on-shell gluons in a non-Abelian $S U\left(N_{c}\right)$ Yang-Mills theory [23,102-105], as in our case. ${ }^{1}$

The previous study [55] mentioned above for a $750 \mathrm{GeV}$ color-singlet spin-0 digluon resonance used parton-level differential cross sections which were then smeared by convolution with an assumed approximate detector response function. Here, we repeat that type of analysis, but also obtain the detector-level dijet invariant-mass distributions for both the signal process alone, and its interference with the QCD background using Monte Carlo event generators including showering and hadronization and detector simulation. The latter method serves as a validation of the qualitative results obtained by the simpler method used in Ref. [55].

The rest of this paper is structured as follows. In Sec. II, we introduce the considered models of various spin and color quantum numbers, along with their effective interaction Lagrangians. To elucidate the importance of the interference effects, we then consider a few benchmark examples for various resonance masses $M_{X}=(750,1000$, $1500,2000,2500,3000) \mathrm{GeV}$, such that their resonant production cross sections are close to the present claimed exclusions of the CMS experiment as given in the most recent reported searches [1,2]. In Sec. III, we discuss the methods and techniques used to obtain smeared partonlevel and full event simulated results. We then present the results for the considered benchmark models in Sec. IV. We start by assuming that $X$ almost always decays to a pair of gluons. We then show in Sec. V that the interference effects are even more dramatic if the digluon resonance has other undetectable decays contributing to its width. (These could include invisible or multijet final states from each $X$ decay.) Finally, in Sec. VI, we conclude the paper by summarizing the significance of the signal/background interference for digluon resonances.

\section{DIGLUON RESONANCES AND BENCHMARK MODELS}

The models considered in this paper are described in the following. In all the models, $X$ is assumed to couple to gluons with nonrenormalizable operators invariant under QCD gauge transformations. The couplings $c_{i}$ are dimensionless, possibly complex form factors, and $\Lambda$ is a mass scale associated with new physics. The effective formfactor couplings will be suppressed by masses of heavier particles, if the interaction is loop induced.

\footnotetext{
${ }^{1}$ The Landau-Yang theorem also rules out the possibility of colored or colorless massive pseudovectors (i.e., even-parity spin-1 particles) decaying to a pair of on-shell massless gauge bosons. In general, this selection rule does not forbid the decay of a massive spin-1 particle to two massless gauge bosons if one of the three bosons is off shell $[104,105]$.
} 


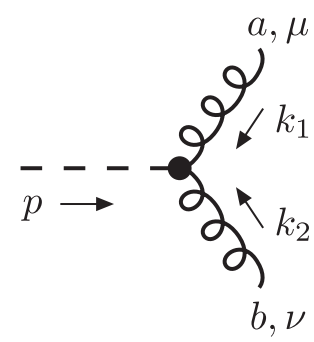

$$
\begin{aligned}
& -i \frac{2 c_{1}}{\Lambda} \delta^{a b}\left(k_{1} \cdot k_{2} \eta^{\mu \nu}-k_{2}^{\mu} k_{1}^{\nu}\right) \quad \text { (scalar) } \\
& -i \frac{2 c_{2}}{\Lambda} \delta^{a b} \epsilon^{\mu \nu \rho \sigma} k_{1 \rho} k_{2 \sigma} \quad \text { (pseudo-scalar) }
\end{aligned}
$$

FIG. 1. Feynman rule for the effective coupling of parity-even (top) and parity-odd (bottom) spin-0 color-singlet resonances with a gluon pair, with $p^{\mu}+k_{1}^{\mu}+k_{2}^{\mu}=0$. Here, $c_{1}$ and $c_{2}$ are dimensionless form factors, and $\Lambda$ is the mass scale associated with the new physics.

Spin 0, color singlet: The effective Lagrangian for an even-parity (scalar) resonance $X$ can be written as

$$
\mathcal{L}=\frac{c_{1}}{2 \Lambda} X F_{\mu \nu}^{a} F^{a \mu \nu},
$$

and for an odd-parity (pseudoscalar) resonance as

$$
\mathcal{L}=-\frac{c_{2}}{4 \Lambda} X \epsilon^{\mu \nu \rho \sigma} F_{\mu \nu}^{a} F_{\rho \sigma}^{a},
$$

where $F_{\mu \nu}^{a}=\partial_{\mu} A_{\nu}^{a}-\partial_{\nu} A_{\mu}^{a}-g_{s} f^{a b c} A_{\mu}^{b} A_{\nu}^{c}$ is the QCD fieldstrength tensor for $a, b, c=1,2, \ldots, 8, f^{a b c}$ are the antisymmetric structure constants of $S U(3)_{c}$, and $g_{s}$ is the strong coupling constant. The corresponding Feynman rules for $X-g-g$ couplings, for both scalar and pseudoscalar color singlets, are shown in Fig. 1.

Spin 0, color octet: The effective Lagrangian for an evenparity (scalar) resonance $X$ is

$$
\mathcal{L}=\frac{c_{3}}{2 \Lambda} d^{a b c} X^{c} F_{\mu \nu}^{a} F^{b \mu \nu},
$$

and for an odd-parity (pseudoscalar) resonance it is

$$
\mathcal{L}=-\frac{c_{4}}{4 \Lambda} d^{a b c} X^{c} \epsilon^{\mu \nu \rho \sigma} F_{\mu \nu}^{a} F_{\rho \sigma}^{b},
$$

where the symmetric anomaly coefficients of $S U(3)_{c}$ are defined as

$$
d^{a b c}=2 \operatorname{Tr}\left[\left\{T^{a}, T^{b}\right\} T^{c}\right]
$$

with the usual normalization for the fundamental representation matrices

$$
\operatorname{Tr}\left[T^{a} T^{b}\right]=\frac{1}{2} \delta^{a b},
$$

so that

$$
d^{a b c} d^{a b e}=\frac{N_{c}^{2}-4}{N_{c}} \delta^{c e}=\frac{5}{3} \delta^{c e} .
$$

The corresponding Feynman rules for the effective couplings of scalar and pseudoscalar color octets with two gluons are shown in Fig. 2.

Spin 1, color octet: The Landau-Yang theorem does not forbid the decay of a color-octet massive vector to an onshell gluon pair [23,102-105]. The effective Lagrangian describing the nontrivial coupling of two gluons to a massive odd-parity, spin-1, color-octet resonance $X_{\mu}^{a}$ is [104]

$$
\mathcal{L}=\frac{c_{5}}{\Lambda^{2}} f^{a b c}\left(D_{\mu} X_{\nu}^{a}-D_{\nu} X_{\mu}^{a}\right) F^{b \nu \rho} F_{\rho}^{c \mu},
$$

where $D_{\mu}$ is the gauge-covariant derivative. (For the $X$-gluon-gluon interaction, only the ordinary partial derivative part of this is pertinent, so one can replace $D_{\mu}$ by $\partial_{\mu}$.) Note that dimensional analysis says that if we want $c_{5}$ to be dimensionless, we now need $\Lambda^{2}$ in the denominator, where $\Lambda$ is the new physics scale. The Feynman rule for a massive color-octet vector coupling to a gluon pair is shown in

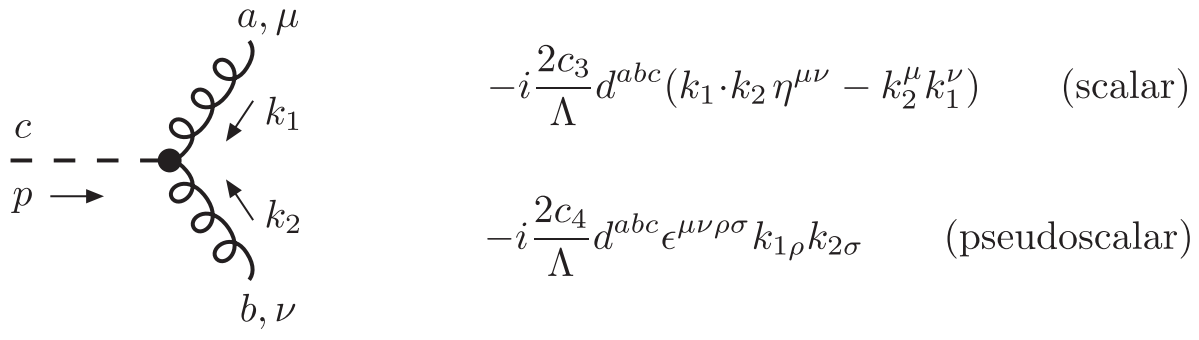

FIG. 2. Feynman rule for the effective coupling of parity-even (top) and parity-odd (bottom) spin-0 color-octet resonances with a gluon pair, with $p^{\mu}+k_{1}^{\mu}+k_{2}^{\mu}=0$. Here, $c_{3}$ and $c_{4}$ are dimensionless form factors, and $\Lambda$ is the mass scale associated with the new physics. 


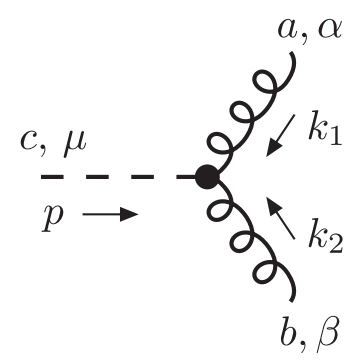

$$
2 i \frac{c_{5}}{\Lambda^{2}} f^{a b c}\left(k_{1}-k_{2}\right)^{\mu}\left(\eta^{\alpha \beta} k_{1} \cdot k_{2}-k_{1}^{\beta} k_{2}^{\alpha}\right)
$$

FIG. 3. Feynman rule for the effective coupling of a spin-1 color-octet resonance with a gluon pair, with $p^{\mu}+k_{1}^{\mu}+k_{2}^{\mu}=0$. Here, $c_{5}$ is a dimensionless form factor, and $\Lambda$ is the mass scale associated with the new physics.

Fig. 3. In the Feynman rule, the contributions proportional to $k_{1}^{\alpha}$ and $k_{2}^{\beta}$ have been dropped, as they never contribute to amplitudes because of $\epsilon_{1} \cdot k_{1}=\epsilon_{2} \cdot k_{2}=0$. It should be noted that our treatment of the spin-1 color octet is not the same as that of the usual "axigluon/coloron" models, as in Refs. [12,20,23]. In those models, the spin-1 color octet appears primarily as a $q \bar{q}$ resonance; although the $g g$ production channel is nonzero, in specific models it is small compared to the $q \bar{q}$ production channel. In this paper, we instead focus on the case that the production of the resonance is mostly through a large coupling to $g g$.

Spin 2, color singlet: The free Lagrangian for a massive spin-2 resonance $X^{\mu \nu}$, first derived by Fierz and Pauli, can be written as [106]

$$
\begin{aligned}
\mathcal{L}_{f}= & \frac{1}{2} X^{\mu \nu} \partial_{\alpha} \partial^{\alpha} X_{\mu \nu}-X^{\mu \nu} \partial_{\mu} \partial^{\alpha} X_{\nu \alpha}+X \partial_{\mu} \partial_{\nu} X^{\mu \nu}-\frac{1}{2} X \partial_{\mu} \partial^{\mu} X \\
& +\frac{1}{2} M_{X}^{2}\left[X^{\mu \nu} X_{\mu \nu}-X^{2}\right],
\end{aligned}
$$

where $X=X^{\alpha}{ }_{\alpha}$.

The effective interaction Lagrangian for an even-parity color-singlet spin-2 resonance $X^{\mu \nu}$ (for example, a KK or RS graviton) can be written as $[48,107,108]$

$$
\mathcal{L}=\frac{1}{\bar{M}_{P}} X^{\mu \nu}\left[F_{\mu \rho}^{a} F_{\nu}^{a \rho}-\frac{1}{4} \eta_{\mu \nu} F_{\rho \sigma}^{a} F^{a \rho \sigma}\right],
$$

where $\bar{M}_{P}$ is a new mass scale. The Feynman rule for the effective coupling of a massive color-singlet spin-2 particle with two gluons is shown in Fig. 4, where

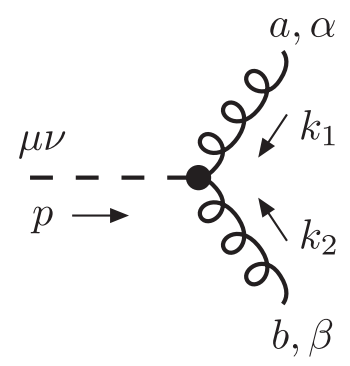

FIG. 4. Feynman rule for the effective coupling of a spin-2 color-singlet resonance with a gluon pair, with $p^{\mu}+k_{1}^{\mu}+k_{2}^{\mu}=0$. Here, $\bar{M}_{P}$ is a new mass scale and $V^{\mu \nu \alpha \beta}$ is defined in Eq. (2.11).

$$
\begin{aligned}
V^{\mu \nu \alpha \beta}= & k_{1} \cdot k_{2}\left(\eta^{\mu \nu} \eta^{\alpha \beta}-\eta^{\mu \alpha} \eta^{\nu \beta}-\eta^{\nu \alpha} \eta^{\mu \beta}\right)-\eta^{\mu \nu} k_{1}^{\beta} k_{2}^{\alpha} \\
& -\left(k_{1}^{\mu} k_{2}^{\nu}+k_{2}^{\mu} k_{1}^{\nu}\right) \eta^{\alpha \beta}+k_{1}^{\mu} k_{2}^{\alpha} \eta^{\nu \beta}+k_{1}^{\nu} k_{2}^{\alpha} \eta^{\mu \beta} \\
& +k_{1}^{\beta} k_{2}^{\nu} \eta^{\mu \alpha}+k_{1}^{\beta} k_{2}^{\mu} \eta^{\nu \alpha} .
\end{aligned}
$$

Note that this satisfies the tracelessness and QCD gauge invariance conditions:

$$
\begin{aligned}
& \eta_{\mu \nu} V^{\mu \nu \alpha \beta}=0, \\
& k_{1 \alpha} V^{\mu \nu \alpha \beta}=0, \\
& k_{2 \beta} V^{\mu \nu \alpha \beta}=0 .
\end{aligned}
$$

One could also consider a theory where the second term (with $\eta_{\mu \nu}$ ) is omitted from the Lagrangian (2.10). In that case, the terms containing $\eta_{\mu \nu}$ are removed from $V^{\mu \nu \alpha \beta}$, and the tracelessness condition is not satisfied. One could also consider a general linear combination of these terms. There are a few other terms that could be written down, involving higher derivatives, but they are omitted from our study.

The propagator for the massive spin- 2 resonance is shown in Fig. 5, where

$$
\begin{gathered}
D_{\mu \nu \rho \sigma}=\frac{1}{2} G_{\mu \rho} G_{\nu \sigma}+\frac{1}{2} G_{\mu \sigma} G_{\nu \rho}-\frac{1}{3} G_{\mu \nu} G_{\rho \sigma}, \\
G_{\mu \nu}=\eta_{\mu \nu}-p_{\mu} p_{\nu} / M_{X}^{2} .
\end{gathered}
$$

The tensor in the numerator of the propagator can be related to a basis for the five symmetric, traceless, orthonormal external state polarization tensors for the spin-2 particle, which can be written as

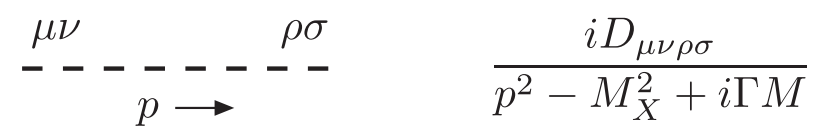

FIG. 5. Propagator for a massive spin-2 resonance. $D_{\mu \nu \rho \sigma}$ is defined in Eq. (2.15). 


$$
\begin{aligned}
\epsilon_{\mu \nu}^{(1)} & =\frac{1}{\sqrt{2}}\left(\begin{array}{llll}
0 & 0 & 0 & 0 \\
0 & 0 & 1 & 0 \\
0 & 1 & 0 & 0 \\
0 & 0 & 0 & 0
\end{array}\right), \\
\epsilon_{\mu \nu}^{(2)} & =\frac{1}{\sqrt{2}}\left(\begin{array}{llll}
0 & 0 & 0 & 0 \\
0 & 0 & 0 & 1 \\
0 & 0 & 0 & 0 \\
0 & 1 & 0 & 0
\end{array}\right), \\
\epsilon_{\mu \nu}^{(3)} & =\frac{1}{\sqrt{2}}\left(\begin{array}{llll}
0 & 0 & 0 & 0 \\
0 & 0 & 0 & 0 \\
0 & 0 & 0 & 1 \\
0 & 0 & 1 & 0
\end{array}\right), \\
\epsilon_{\mu \nu}^{(4)} & =\frac{1}{\sqrt{2}}\left(\begin{array}{cccc}
0 & 0 & 0 & 0 \\
0 & 1 & 0 & 0 \\
0 & 0 & -1 & 0 \\
0 & 0 & 0 & 0
\end{array}\right), \\
\epsilon_{\mu \nu}^{(5)} & =\frac{1}{\sqrt{6}}\left(\begin{array}{llll}
0 & 0 & 0 & 0 \\
0 & 1 & 0 & 0 \\
0 & 0 & 1 & 0 \\
0 & 0 & 0 & -2
\end{array}\right) .
\end{aligned}
$$

These satisfy

$$
\epsilon_{\mu \nu}^{(i)} \epsilon^{(j) \mu \nu}=\delta^{i j} \quad(i, \quad j=1, \ldots, 5),
$$

and

$$
\sum_{i=1}^{5} \epsilon_{\mu \nu}^{(i)} \epsilon_{\rho \sigma}^{(i)}=D_{\mu \nu \rho \sigma}
$$

which imply

$$
\epsilon_{\mu \nu}^{(i)} D^{\mu \nu \rho \sigma}=\epsilon^{(i) \rho \sigma} \quad(i=1, \ldots, 5) .
$$

For simplicity, we consider here only the case that the spin-2 resonance coupling to two partons is only (or mainly) to two gluons. A KK or RS graviton would also couple to $q \bar{q}$, which would result in a more complicated analysis. The interference effect would be smaller in those cases relative to the resonant dijet process, because the $q \bar{q}$ initial and final states for $X$ production and decay of course do not interfere with the large $g g \rightarrow g g$ QCD amplitude.

We chose benchmark models as specified in Table I such that the $s$-channel resonant-only cross sections are close to the claimed exclusions by CMS in Refs. [1,2]. Specifically, for the resonance masses $M_{X}=(750,1000,1500,2000$, 2500,3000) $\mathrm{GeV}$, the $95 \%$ C.L. upper limits observed by CMS in Refs. [1,2] are $(5.5,1.66,0.42,0.22837,0.032155$,
TABLE I. Our choice of benchmark masses and widths for the digluon resonances in four $(J=$ spin, color) representations considered in this paper. Here, $M_{X}, \Gamma$, and $\Gamma_{g g}$ are the mass, total width, and digluon partial width of $X$, respectively.

\begin{tabular}{lllll}
\hline \hline $\begin{array}{l}\text { Resonance } \\
\text { Mass } M_{X}\end{array}$ & \multicolumn{4}{c}{$\Gamma_{g g}^{2} / \Gamma M_{X}$} \\
\cline { 2 - 5 }$(\mathrm{GeV})$ & $\begin{array}{l}J=0, \\
\text { singlet }\end{array}$ & $\begin{array}{c}J=0, \\
\text { octet }\end{array}$ & $\begin{array}{c}J=1, \\
\text { octet }\end{array}$ & $\begin{array}{l}J=2, \\
\text { singlet }\end{array}$ \\
\hline 750 & 0.0015 & 0.00016 & 0.00005 & 0.0003 \\
1000 & 0.002 & 0.0002 & 0.000065 & 0.00041 \\
1500 & 0.005 & 0.0005 & 0.00015 & 0.001 \\
2000 & 0.019 & 0.0018 & 0.00054 & 0.00375 \\
2500 & 0.0108 & 0.001 & 0.0003 & 0.0022 \\
3000 & 0.07 & 0.006 & 0.00183 & 0.014 \\
\hline \hline
\end{tabular}

0.043386) pb. In Table I, $\Gamma_{g g}^{2} / \Gamma M_{X}$ increases as we move to higher masses, except from $M_{X}=2000-2500 \mathrm{GeV}$; this is because the observed limit at $M_{X}=2500 \mathrm{GeV}$ is evidently a downward fluctuation compared to the limits obtained at $M_{X}=2000$ and $3000 \mathrm{GeV}$. For the $s$-channel resonant cross sections, we chose a $K$ factor of 1.5 and an acceptance of 0.5 for the purposes of choosing the benchmarks. The claimed limits as a function of mass, and the predicted resonance cross sections for an example $M_{X}=2000 \mathrm{GeV}$, are shown in Fig. 6. For our first set of studies below, we chose the digluon partial width of $X$ (denoted in this paper as $\Gamma_{g g}$ ) to be the same as the total width $(\Gamma)$. More generally, if $X$ can decay to final states that are more difficult to detect for some reason or are just not part of the dijet search (for example, $X \rightarrow j j j, X \rightarrow j j j j$, or $X \rightarrow$ invisibles), then the total width $\Gamma$ might exceed $\Gamma_{g g}$, and $\mathrm{BR}(X \rightarrow g g)=\Gamma_{g g} / \Gamma$.

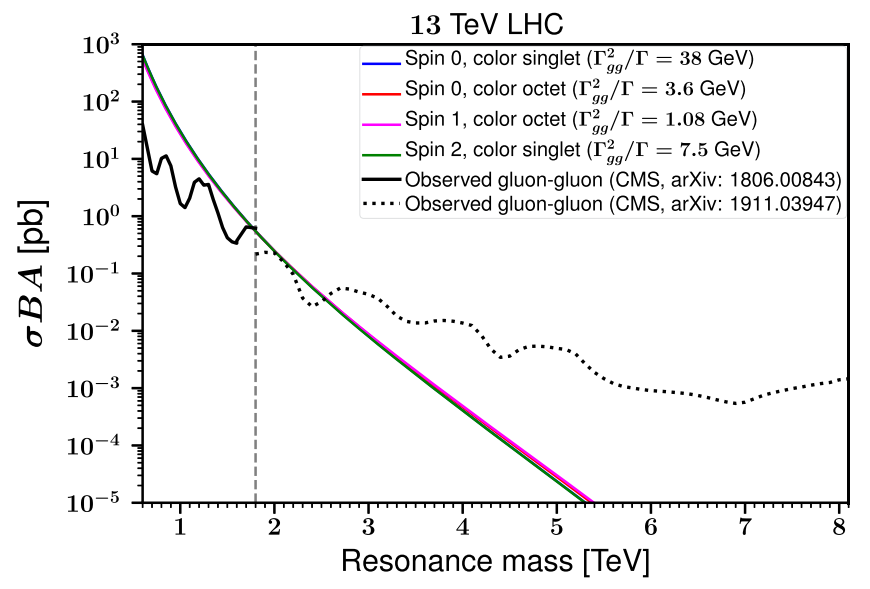

FIG. 6. The observed 95\% C.L. upper limits on the product of the cross section $(\sigma)$, branching fraction $(B)$, and acceptance $(A)$ for dijet resonances decaying to a gluon pair from Ref. [2] (solid black line, left of vertical dashed gray line) and Ref. [1] (dotted black line, right of vertical dashed gray line). The solid colored lines show the leading-order $s$-channel resonant cross sections, in the narrow-width approximation, for the benchmarks for a resonance mass of $2000 \mathrm{GeV}$ in each of the considered models of digluon resonances. 
The resonance partonic total cross section (after angular integration, and with no cuts) in the narrow-width approximation is equal to

$\hat{\sigma}(g g \rightarrow X \rightarrow g g)=(2 j+1) k \frac{\Gamma_{g g}^{2}}{\Gamma M_{X}} \frac{\pi^{2}}{4} \delta\left(\hat{s}-M_{X}^{2}\right)$,

where $j=0,1,2$ and $k=1,8$ are the spin and color of $X$, and $\sqrt{\hat{s}}$ is the partonic invariant mass. This can be checked as a limit in each of the special-case results of the next section, and reflects the fact that there are $(2 j+1) k$ times more spin $\otimes$ color states for a spin- $j$, color- $k$ resonance than for a spin-0 color singlet. Therefore, for our models with $j \neq 0$ and/or $k \neq 1$, we chose benchmark points that have $\Gamma_{g g}^{2} / \Gamma$ approximately $(2 j+1) k$ times smaller than for a spin- 0 color singlet of the same mass, in order to maintain (roughly) the same resonant total cross section.

\section{SIGNAL-BACKGROUND INTERFERENCE: PARTON-LEVEL AND MONTE CARLO METHODS}

\section{A. Parton-level approximation}

For both the resonant signal $g g \rightarrow X \rightarrow g g$ and the continuum QCD background $g g \rightarrow g g$ processes, we can write an amplitude, for each choice of external gluon polarizations, in terms of a redundant basis of color combinations:

$$
\begin{aligned}
\mathcal{A}^{a b c d}= & a_{1} f^{a b e} f^{c d e}+a_{2} f^{a c e} f^{b d e}+a_{3} f^{a d e} f^{b c e}+a_{4} \delta^{a b} \delta^{c d}+a_{5} \delta^{a c} \delta^{b d}+a_{6} \delta^{a d} \delta^{b c} \\
& +a_{7} d^{a b e} d^{c d e}+a_{8} d^{a c e} d^{b d e}+a_{9} d^{a d e} d^{b c e}
\end{aligned}
$$

which then leads to the color sum

$$
\begin{aligned}
\sum_{a, b, c, d}\left|\mathcal{A}^{a b c d}\right|^{2}= & 72\left(\left|a_{1}\right|^{2}+\left|a_{2}\right|^{2}+\left|a_{3}\right|^{2}\right)+72 \operatorname{Re}\left[a_{1} a_{2}^{*}-a_{1} a_{3}^{*}+a_{2} a_{3}^{*}\right] \\
& +64\left(\left|a_{4}\right|^{2}+\left|a_{5}\right|^{2}+\left|a_{6}\right|^{2}\right)+16 \operatorname{Re}\left[a_{4} a_{5}^{*}+a_{4} a_{6}^{*}+a_{5} a_{6}^{*}\right] \\
& +\frac{200}{9}\left(\left|a_{7}\right|^{2}+\left|a_{8}\right|^{2}+\left|a_{9}\right|^{2}\right)-\frac{40}{3} \operatorname{Re}\left[a_{7} a_{8}^{*}+a_{7} a_{9}^{*}+a_{8} a_{9}^{*}\right] \\
& +48 \operatorname{Re}\left[a_{1} a_{5}^{*}-a_{1} a_{6}^{*}+a_{2} a_{4}^{*}-a_{2} a_{6}^{*}+a_{3} a_{4}^{*}-a_{3} a_{5}^{*}\right] \\
& +40 \operatorname{Re}\left[a_{1} a_{8}^{*}-a_{1} a_{9}^{*}+a_{2} a_{7}^{*}-a_{2} a_{9}^{*}+a_{3} a_{7}^{*}-a_{3} a_{8}^{*}\right] \\
& +\frac{80}{3} \operatorname{Re}\left[a_{4} a_{8}^{*}+a_{4} a_{9}^{*}+a_{5} a_{7}^{*}+a_{5} a_{9}^{*}+a_{6} a_{7}^{*}+a_{6} a_{8}^{*}\right] .
\end{aligned}
$$

Of the nine coefficients $a_{i}$ in Eq. (3.1), only five are independent. This redundancy is reflected in the following QCD identities $[109,110]$ :

$$
\begin{gathered}
f^{a b e} f^{c d e}+f^{a c e} f^{d b e}+f^{a d e} f^{b c e}=0, \\
d^{a b e} d^{c d e}-\frac{1}{3}\left(f^{a c e} f^{b d e}+f^{a d e} f^{b c e}\right)=\frac{1}{3}\left(\delta^{a c} \delta^{b d}+\delta^{a d} \delta^{b c}-\delta^{a b} \delta^{c d}\right), \\
f^{a b e} f^{c d e}-d^{a c e} d^{b d e}+d^{b c e} d^{a d e}=\frac{2}{N_{c}}\left(\delta^{a c} \delta^{b d}-\delta^{a d} \delta^{b c}\right), \\
d^{a b e} d^{c d e}+d^{a c e} d^{b d e}+d^{a d e} d^{b c e}=\frac{1}{3}\left(\delta^{a b} \delta^{c d}+\delta^{a c} \delta^{b d}+\delta^{a d} \delta^{b c}\right),
\end{gathered}
$$

where Eq. (3.3) is the Jacobi identity, which holds true for the structure constants of any $S U\left(N_{c}\right)$ group. Equation (3.5) also holds true for any $S U\left(N_{c}\right)$. On the other hand, the identities in Eqs. (3.4) and (3.6) are unique to $S U(3)$. It should be noted that the above four identities are not independent of each other. Namely, Eq. (3.4) is obtained by using Eq. (3.5) in conjunction with Eq. (3.6).
Also, Eq. (3.4) along with Eq. (3.5) gives Eqs. (3.3) and (3.6). Using Eqs. (3.3) and (3.4), we can eliminate the coefficients $a_{7}, a_{8}, a_{9}$, and one of $a_{1}, a_{2}$, or $a_{3}$ in Eq. (3.1). Or we can use Eqs. (3.5) and (3.6) to eliminate the coefficients $a_{1}, a_{2}, a_{3}$, and one of $a_{7}, a_{8}$, or $a_{9}$. In any case, we can eliminate four out of the nine coefficients $a_{i}$ in Eq. (3.1). 
Although the QCD identities in Eqs. (3.3)-(3.6) can be used to rearrange the coefficients, there are natural choices that follow from the Feynman rules. After including the contributions from $t$ - and $u$-channel exchanges of $X$, and the interferences with QCD amplitudes, for color-singlet digluon resonances one naturally has $a_{7}=a_{8}=a_{9}=0$. For coloroctet digluon resonances with spin 0 , one has $a_{4}=a_{5}=$ $a_{6}=0$. For color-octet digluon resonances with spin 1 , one has $a_{4}=a_{5}=a_{6}=a_{7}=a_{8}=a_{9}=0$ in Eq. (3.1),

Equation (3.2), which is understood to also include the interference terms, can then be summed over all of the final states, and averaged over all of the initial states for both color and spin to finally obtain the complete LO partonic differential cross section of all $X$ exchange diagrams (signal), pure QCD background, and the interference between the signal and the background processes. We then define

$$
\begin{aligned}
\frac{d \hat{\sigma}}{d z}= & \frac{d \hat{\sigma}_{s}}{d z}+\frac{d \hat{\sigma}_{t}}{d z}+\frac{d \hat{\sigma}_{u}}{d z}+\frac{d \hat{\sigma}_{s, t}}{d z}+\frac{d \hat{\sigma}_{t, u}}{d z}+\frac{d \hat{\sigma}_{s, u}}{d z} \\
& +\frac{d \hat{\sigma}_{s, \mathrm{QCD}}}{d z}+\frac{d \hat{\sigma}_{t, \mathrm{QCD}}}{d z}+\frac{d \hat{\sigma}_{u, \mathrm{QCD}}}{d z},
\end{aligned}
$$

excluding the pure QCD contribution. In the above definition, $d \hat{\sigma}_{s, \mathrm{QCD}} / d z$, for example, stands for the interference of the $s$-channel $X$ exchange diagram with the pure QCD amplitude. Also, $z$ is the cosine of the gluon scattering angle in the partonic center-of-momentum frame. Analytic formulas for all of the components of $d \hat{\sigma} / d z$ as defined in Eq. (3.7), for each of the digluon resonances considered, are listed next.

Spin 0, color singlet: The resonant and interference partonic cross sections at leading order for both parity-even and parity-odd spin- 0 color singlets (i.e., $i=1,2$ ) are

$$
\begin{gathered}
\frac{d \hat{\sigma}_{s}}{d z}=\frac{\left|c_{i}\right|^{4} \hat{s}^{3}}{32 \pi \Lambda^{4} D(\hat{s})} \\
\frac{d \hat{\sigma}_{t}}{d z}+\frac{d \hat{\sigma}_{u}}{d z}=\frac{\left|c_{i}\right|^{4}}{32 \pi \Lambda^{4} \hat{s}}\left[\frac{\hat{t}^{4}}{D(\hat{t})}+\frac{\hat{u}^{4}}{D(\hat{u})}\right] \\
\frac{d \hat{\sigma}_{s, t}}{d z}=\frac{\left|c_{i}\right|^{4} \hat{s} \hat{t}^{2}}{256 \pi \Lambda^{4} D(\hat{s}) D(\hat{t})}\left[\left(\hat{s}-M_{X}^{2}\right)\left(\hat{t}-M_{X}^{2}\right)+\Gamma^{2} M_{X}^{2}\right]
\end{gathered}
$$

$$
\frac{d \hat{\sigma}_{t, u}}{d z}=\frac{\left|c_{i}\right|^{4} \hat{t}^{2} \hat{u}^{2}}{256 \pi \Lambda^{4} \hat{s} D(\hat{t}) D(\hat{u})}\left[\left(\hat{t}-M_{X}^{2}\right)\left(\hat{u}-M_{X}^{2}\right)+\Gamma^{2} M_{X}^{2}\right],
$$

$$
\frac{d \hat{\sigma}_{s, u}}{d z}=\frac{\left|c_{i}\right|^{4} \hat{s} \hat{u}^{2}}{256 \pi \Lambda^{4} D(\hat{s}) D(\hat{u})}\left[\left(\hat{s}-M_{X}^{2}\right)\left(\hat{u}-M_{X}^{2}\right)+\Gamma^{2} M_{X}^{2}\right],
$$

$$
\begin{aligned}
\frac{d \hat{\sigma}_{s, \mathrm{QCD}}}{d z}= & -\frac{3 \alpha_{S} \hat{s}}{8 \Lambda^{2} D(\hat{s})\left(1-z^{2}\right)} \\
& \times\left\{\operatorname{Re}\left[c_{i}^{2}\right]\left(\hat{s}-M_{X}^{2}\right)+\operatorname{Im}\left[c_{i}^{2}\right] \Gamma M_{X}\right\}, \\
\frac{d \hat{\sigma}_{t, \mathrm{QCD}}}{d z}= & \frac{3 \alpha_{S} \hat{s}(1-z)^{4}}{256 \Lambda^{2} D(\hat{t})(1+z)} \\
& \times\left\{\operatorname{Re}\left[c_{i}^{2}\right]\left(\hat{t}-M_{X}^{2}\right)+\operatorname{Im}\left[c_{i}^{2}\right] \Gamma M_{X}\right\}, \\
\frac{d \hat{\sigma}_{u, \mathrm{QCD}}}{d z}= & \frac{3 \alpha_{S} \hat{s}(1+z)^{4}}{256 \Lambda^{2} D(\hat{u})(1-z)} \\
& \times\left\{\operatorname{Re}\left[c_{i}^{2}\right]\left(\hat{u}-M_{X}^{2}\right)+\operatorname{Im}\left[c_{i}^{2}\right] \Gamma M_{X}\right\},
\end{aligned}
$$

where $\hat{t}=\hat{s}(z-1) / 2$ and $\hat{u}=-\hat{s}(z+1) / 2$, and

$$
D(\hat{s}) \equiv\left(\hat{s}-M_{X}^{2}\right)^{2}+\Gamma^{2} M_{X}^{2} .
$$

Also, the LO partial width into the digluon final state is

$$
\Gamma_{g g}=\frac{\left|c_{i}\right|^{2} M_{X}^{3}}{2 \pi \Lambda^{2}} .
$$

Spin 0, color octet: The resonant and interference partonic cross sections at leading order for both scalar and pseudoscalar color octets (i.e., $i=3,4$ ) are

$$
\frac{d \hat{\sigma}_{s}}{d z}=\frac{25\left|c_{i}\right|^{4} \hat{s}^{3}}{2304 \pi \Lambda^{4} D(\hat{s})},
$$

$$
\frac{d \hat{\sigma}_{t}}{d z}+\frac{d \hat{\sigma}_{u}}{d z}=\frac{25\left|c_{i}\right|^{4}}{2304 \pi \Lambda^{4} \hat{s}}\left[\frac{\hat{t}^{4}}{D(\hat{t})}+\frac{\hat{u}^{4}}{D(\hat{u})}\right]
$$

$$
\frac{d \hat{\sigma}_{s, t}}{d z}=-\frac{5\left|c_{i}\right|^{4} \hat{s} \hat{t}^{2}}{1536 \pi \Lambda^{4} D(\hat{s}) D(\hat{t})}\left[\left(\hat{s}-M_{X}^{2}\right)\left(\hat{t}-M_{X}^{2}\right)+\Gamma^{2} M_{X}^{2}\right],
$$

$$
\frac{d \hat{\sigma}_{t, u}}{d z}=-\frac{5\left|c_{i}\right|^{4} \hat{t}^{2} \hat{u}^{2}}{1536 \pi \Lambda^{4} \hat{s} D(\hat{t}) D(\hat{u})}\left[\left(\hat{t}-M_{X}^{2}\right)\left(\hat{u}-M_{X}^{2}\right)+\Gamma^{2} M_{X}^{2}\right],
$$

$$
\frac{d \hat{\sigma}_{s, u}}{d z}=-\frac{5\left|c_{i}\right|^{4} \hat{s} \hat{u}^{2}}{1536 \pi \Lambda^{4} D(\hat{s}) D(\hat{u})}\left[\left(\hat{s}-M_{X}^{2}\right)\left(\hat{u}-M_{X}^{2}\right)+\Gamma^{2} M_{X}^{2}\right],
$$

$$
\begin{aligned}
\frac{d \hat{\sigma}_{s, \mathrm{QCD}}}{d z}= & -\frac{5 \alpha_{S} \hat{s}}{16 \Lambda^{2} D(\hat{s})\left(1-z^{2}\right)} \\
& \times\left\{\operatorname{Re}\left[c_{i}^{2}\right]\left(\hat{s}-M_{X}^{2}\right)+\operatorname{Im}\left[c_{i}^{2}\right] \Gamma M_{X}\right\}, \\
\frac{d \hat{\sigma}_{t, \mathrm{QCD}}}{d z}= & \frac{5 \alpha_{S} \hat{s}(1-z)^{4}}{512 \Lambda^{2} D(\hat{t})(1+z)} \\
& \times\left\{\operatorname{Re}\left[c_{i}^{2}\right]\left(\hat{t}-M_{X}^{2}\right)+\operatorname{Im}\left[c_{i}^{2}\right] \Gamma M_{X}\right\},
\end{aligned}
$$




$$
\begin{aligned}
\frac{d \hat{\sigma}_{u, \mathrm{QCD}}}{d z}= & \frac{5 \alpha_{S} \hat{s}(1+z)^{4}}{512 \Lambda^{2} D(\hat{u})(1-z)} \\
& \times\left\{\operatorname{Re}\left[c_{i}^{2}\right]\left(\hat{u}-M_{X}^{2}\right)+\operatorname{Im}\left[c_{i}^{2}\right] \Gamma M_{X}\right\},
\end{aligned}
$$

and the partial width into the digluon final state is

$$
\Gamma_{g g}=\frac{5\left|c_{i}\right|^{2} M_{X}^{3}}{48 \pi \Lambda^{2}} .
$$

In the case that the decay width is entirely due to digluons, we can compare the $s$-channel resonant production cross section to that of the spin-0, color-singlet case, using

$$
\frac{1}{D(\hat{s})}=\frac{\pi}{\Gamma M_{X}} \delta\left(\hat{s}-M_{X}^{2}\right)
$$

This means that the $s$-channel resonant cross sections can be written as

$$
\frac{d \hat{\sigma}_{s}}{d z}=n_{i}^{s} \frac{\pi\left|c_{i}\right|^{2} M_{X}^{2}}{\Lambda^{2}} \delta\left(\hat{s}-M_{X}^{2}\right),
$$

where for spin- 0 color singlets

$$
n_{1}^{s}=n_{2}^{s}=1 / 16
$$

and for spin- 0 color octets

$$
n_{3}^{s}=n_{4}^{s}=5 / 48
$$

To have the same resonant cross section, we could take $\left|c_{3}\right|^{2}=(3 / 5)\left|c_{1}\right|^{2}$. In that case, comparing Eqs. (3.13)(3.15) and (3.23)-(3.25), we see that the prefactor for the interference cross section would be half as big for the coloroctet case as for the color-singlet case. So, all other things being equal, the importance of the interference terms relative to the resonance terms is half as big in the color-octet spin- 0 case as in the color-singlet spin- 0 case.

Spin 1, color octet: The resonant and interference partonic cross sections at leading order for a spin-1 color-octet resonance are

$$
\begin{gathered}
\frac{d \hat{\sigma}_{s}}{d z}=\frac{9\left|c_{5}\right|^{4} \hat{s}^{5} z^{2}}{256 \pi \Lambda^{8} D(\hat{s})} \\
\frac{d \hat{\sigma}_{t}}{d z}+\frac{d \hat{\sigma}_{u}}{d z}=\frac{9\left|c_{5}\right|^{4} \hat{s}}{1024 \pi \Lambda^{8}}\left[\frac{\hat{t}^{4}(3+z)^{2}}{D(\hat{t})}+\frac{\hat{u}^{4}(3-z)^{2}}{D(\hat{u})}\right] \\
\frac{d \hat{\sigma}_{s, t}}{d z}=\frac{9\left|c_{5}\right|^{4} \hat{s}^{3} \hat{t}^{2} z(3+z)}{1024 \pi \Lambda^{8} D(\hat{s}) D(\hat{t})}\left[\left(\hat{s}-M_{X}^{2}\right)\left(\hat{t}-M_{X}^{2}\right)+\Gamma^{2} M_{X}^{2}\right]
\end{gathered}
$$

$$
\frac{d \hat{\sigma}_{t, u}}{d z}=\frac{9\left|c_{5}\right|^{4} \hat{s}^{2} \hat{u}^{2}\left(9-z^{2}\right)}{2048 \pi \Lambda^{8} D(\hat{t}) D(\hat{u})}\left[\left(\hat{t}-M_{X}^{2}\right)\left(\hat{u}-M_{X}^{2}\right)+\Gamma^{2} M_{X}^{2}\right],
$$

$$
\frac{d \hat{\sigma}_{s, u}}{d z}=-\frac{9\left|c_{5}\right|^{4} \hat{s}^{3} \hat{u}^{2} z(3-z)}{1024 \pi \Lambda^{8} D(\hat{s}) D(\hat{u})}\left[\left(\hat{s}-M_{X}^{2}\right)\left(\hat{u}-M_{X}^{2}\right)+\Gamma^{2} M_{X}^{2}\right],
$$

$$
\begin{aligned}
\frac{d \hat{\sigma}_{s, \mathrm{QCD}}}{d z}= & -\frac{9 \alpha_{S} \hat{s}^{2} z^{2}}{16 \Lambda^{4} D(\hat{s})\left(1-z^{2}\right)} \\
& \times\left\{\operatorname{Re}\left[c_{5}^{2}\right]\left(\hat{s}-M_{X}^{2}\right)+\operatorname{Im}\left[c_{5}^{2}\right] \Gamma M_{X}\right\}
\end{aligned}
$$

$$
\begin{aligned}
\frac{d \hat{\sigma}_{t, \mathrm{QCD}}}{d z}= & -\frac{9 \alpha_{S} \hat{s}^{2}(3+z)^{2}(1-z)^{3}}{1024 \Lambda^{4} D(\hat{t})(1+z)} \\
& \times\left\{\operatorname{Re}\left[c_{5}^{2}\right]\left(\hat{t}-M_{X}^{2}\right)+\operatorname{Im}\left[c_{5}^{2}\right] \Gamma M_{X}\right\},
\end{aligned}
$$

$$
\begin{aligned}
\frac{d \hat{\sigma}_{u, \mathrm{QCD}}}{d z}= & -\frac{9 \alpha_{S} \hat{s}^{2}(3-z)^{2}(1+z)^{3}}{1024 \Lambda^{4} D(\hat{u})(1-z)} \\
& \times\left\{\operatorname{Re}\left[c_{5}^{2}\right]\left(\hat{u}-M_{X}^{2}\right)+\operatorname{Im}\left[c_{5}^{2}\right] \Gamma M_{X}\right\},
\end{aligned}
$$

and the partial width into the digluon final state is

$$
\Gamma_{g g}=\frac{\left|c_{5}\right|^{2} M_{X}^{5}}{16 \pi \Lambda^{4}}
$$

Spin 2, color singlet: The resonant and interference partonic cross sections at leading order for a spin-2 colorsinglet resonance are

$$
\begin{gathered}
\frac{d \hat{\sigma}_{s}}{d z}=\frac{\hat{s}^{3}\left(1+6 z^{2}+z^{4}\right)}{512 \pi \bar{M}^{4}{ }_{P} D(\hat{s})}, \\
\frac{d \hat{\sigma}_{t}}{d z}=\frac{\hat{s}^{3}\left(17+4 z+6 z^{2}+4 z^{3}+z^{4}\right)}{1024 \pi \bar{M}^{4}{ }_{P} D(\hat{t})}, \\
\frac{d \hat{\sigma}_{u}}{d z}=\frac{\hat{s}^{3}\left(17-4 z+6 z^{2}-4 z^{3}+z^{4}\right)}{1024 \pi \bar{M}^{4}{ }_{P} D(\hat{u})},
\end{gathered}
$$

$$
\frac{d \hat{\sigma}_{s, t}}{d z}=\frac{\hat{s}^{3}(1+z)^{4}}{4096 \pi \bar{M}^{4}{ }_{P} D(\hat{s}) D(\hat{t})}\left[\left(\hat{s}-M_{X}^{2}\right)\left(\hat{t}-M_{X}^{2}\right)+\Gamma^{2} M_{X}^{2}\right],
$$

$$
\frac{d \hat{\sigma}_{t, u}}{d z}=\frac{\hat{s}^{3}}{256 \pi \bar{M}^{4}{ }_{P} D(\hat{t}) D(\hat{u})}\left[\left(\hat{t}-M_{X}^{2}\right)\left(\hat{u}-M_{X}^{2}\right)+\Gamma^{2} M_{X}^{2}\right],
$$

$$
\frac{d \hat{\sigma}_{s, u}}{d z}=\frac{\hat{s}^{3}(1-z)^{4}}{4096 \pi \bar{M}^{4}{ }_{P} D(\hat{s}) D(\hat{u})}\left[\left(\hat{s}-M_{X}^{2}\right)\left(\hat{u}-M_{X}^{2}\right)+\Gamma^{2} M_{X}^{2}\right],
$$




$$
\begin{gathered}
\frac{d \hat{\sigma}_{s, \mathrm{QCD}}}{d z}=-\frac{3 \alpha_{S} \hat{s}\left(\hat{s}-M_{X}^{2}\right)\left(1+6 z^{2}+z^{4}\right)}{64 \bar{M}^{2}{ }_{P} D(\hat{s})\left(1-z^{2}\right)}, \\
\frac{d \hat{\sigma}_{t, \mathrm{QCD}}}{d z}=\frac{3 \alpha_{S} \hat{s}\left(\hat{t}-M_{X}^{2}\right)\left(17+4 z+6 z^{2}+4 z^{3}+z^{4}\right)}{256 \bar{M}^{2}{ }_{P} D(\hat{t})\left(1-z^{2}\right)}, \\
\frac{d \hat{\sigma}_{u, \mathrm{QCD}}}{d z}=\frac{3 \alpha_{S} \hat{s}\left(\hat{u}-M_{X}^{2}\right)\left(17-4 z+6 z^{2}-4 z^{3}+z^{4}\right)}{256 \bar{M}^{2}{ }_{P} D(\hat{u})\left(1-z^{2}\right)} .
\end{gathered}
$$

The pure $X$-exchange parts and their corresponding interference-with-QCD terms have common angular factors of $\left(1+6 z^{2}+z^{4}\right),\left(17+4 z+6 z^{2}+4 z^{3}+z^{4}\right)$, and $\left(17-4 z+6 z^{2}-4 z^{3}+z^{4}\right)$ for $s, t$, and $u$ channels respectively.

The digluon partial width of the resonance is

$$
\Gamma_{g g}=\frac{M_{X}^{3}}{10 \pi \bar{M}_{P}^{2}} .
$$

In the narrow-width approximation of Eq. (3.27), assuming that $X$ has no other decays, we therefore have

$$
\frac{d \hat{\sigma}_{s}}{d z}=\frac{5 \pi M_{X}^{2}}{256 \bar{M}_{P}^{2}}\left(1+6 z^{2}+z^{4}\right) \delta\left(\hat{s}-M_{X}^{2}\right) .
$$

Having obtained the analytic formulas for all of the components of $d \hat{\sigma} / d z$, for all four models considered, we can compute the $\mathrm{LO}$ digluon production cross section at the LHC,

$\frac{d \sigma_{p p \rightarrow g g}}{d(\sqrt{\hat{s}})}=\sqrt{\hat{s}} \int_{x_{-}}^{x_{+}} \frac{d x}{x s} g(x) g\left(\frac{\hat{s}}{x s}\right) \int_{-z_{\text {cut }}}^{z_{\mathrm{cut}}} d z \frac{d \hat{\sigma}}{d z}$.

Here, $x$ is the longitudinal momentum fraction for the parton, $g(x)$ is the gluon parton distribution function (PDF) obtained from the NNPDF2.3 LO PDF set [111] with factorization scale $\mu_{F}=M_{X}, \sqrt{s}$ is the total energy of the $p p$ collisions at the LHC, and $\sqrt{\hat{s}}$ is the invariant mass of the gluon pairs in both initial and final states. In Eq. (3.51), we have imposed cuts on the transverse momentum and the pseudorapidity of the gluons:

$$
\begin{gathered}
p_{T_{j}}>p_{T_{j}}^{\text {cut }}=100 \mathrm{GeV}, \\
\left|\eta_{j}\right|<\eta_{j}^{\text {cut }}=2.5,
\end{gathered}
$$

respectively. Also, in order to increase the significance of the resonance signal, CMS has defined signal regions that cut on the difference between the pseudorapidities of the two jets:

$$
\Delta \eta=\left|\eta_{j_{1}}-\eta_{j_{2}}\right|<(\Delta \eta)^{\mathrm{cut}} .
$$

We will follow CMS by choosing $(\Delta \eta)^{\text {cut }}=1.1$ for $M_{X}>$ $1800 \mathrm{GeV}$ as in Ref. [2], and $(\Delta \eta)^{\text {cut }}=1.3$ for $M_{X}<$ $1800 \mathrm{GeV}$ as in Ref. [1]. For a parton-level analysis, these cuts can be imposed by using

$$
\begin{gathered}
x_{ \pm}=e^{ \pm \eta_{j}^{\text {cut }}} \sqrt{\hat{s} / s} \\
z_{\text {cut }}=\operatorname{Min}\left[\sqrt{1-4 p_{T_{j}}^{\text {cut }^{2}} / \hat{s}}, \tanh \left(\eta_{j}^{\text {cut }}-\frac{1}{2}\left|\ln \left(x^{2} s / \hat{s}\right)\right|\right)\right. \\
\left.\tanh \left((\Delta \eta)^{\text {cut }} / 2\right)\right]
\end{gathered}
$$

in Eq. (3.51).

Then, to obtain more realistic distributions roughly approximating the experimental ones, the parton-level distributions of $d \sigma_{p p \rightarrow g g} / d(\sqrt{\hat{s}})$ in Eq. (3.51) can be smeared by convolution with an approximate detector response dijet mass distribution, shown for the case of a digluon invariant mass $m_{g g}=(1000,2000,3000) \mathrm{GeV}$ in Fig. 7. To obtain the approximate detector responses for the dijet invariant-mass distributions, we used a sample of detector-level events, generated with PYTHIA8.2 [112,113] and DELPHES3.4 [114], obtained from (at least $1.5 \times 10^{7}$ ) parton-level events for the resonant process $g g \rightarrow X \rightarrow g g$ with the required digluon invariant mass (here $\sqrt{\hat{s}}=m_{g g}=$ 1000,2000 , and $3000 \mathrm{GeV}$ ) using the same cuts and procedure for combining jets into wide jets as described in the next subsection. This was done for each of the four (spin, color) quantum number combinations described above.

The results obtained by the above simple procedure will be referred to below as the parton-level approximation (with smearing). This method can be viewed as a quick

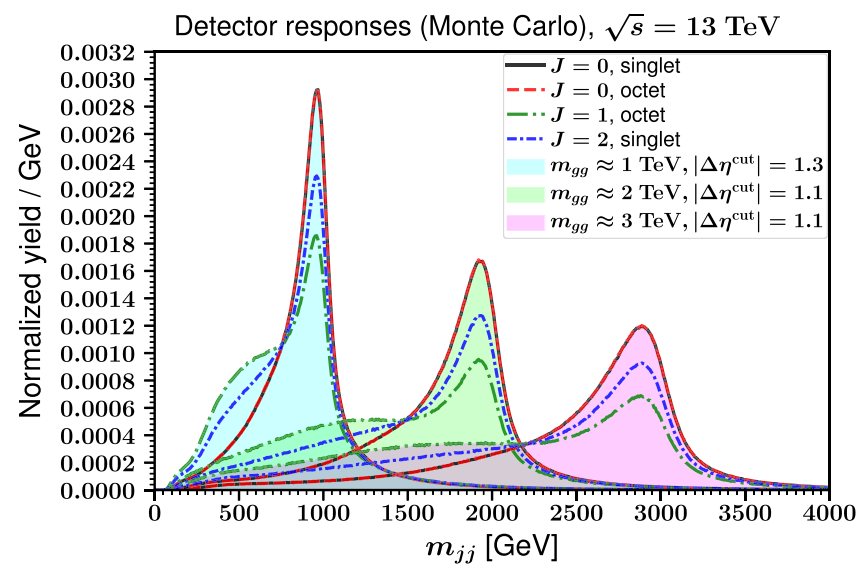

FIG. 7. The normalized detector response dijet mass distributions for two wide jets, for digluon masses $m_{g g}=1000,2000$, and $3000 \mathrm{GeV}$, and various spin and color quantum numbers, generated and analyzed using PYTHIA8 and DELPHES. 
approximation and qualitative independent cross-check of the more involved method we describe next, which is much more computationally demanding.

\section{B. Showering, hadronization, and detector-level simulation}

For a much more realistic approximation, referred to below simply as the detector-level simulation, we used MADGRAPH5_aMC@NLOv2.6.6 [115] for all of our LO event simulations, which uses a Universal FeynRules Output file generated by FeynRulesv2.3 [116], a Mathematica package to get the Feynman rules from an input Lagrangian. We used PYTHIA8.2 for showering and hadronization, and DELPHES3.4 for detector simulation.

Using MADGRAPH, our goal was to generate detectorlevel events not only for the signal, including all $X$ exchange diagrams, but also for the interference between the signal and the QCD background $g g \rightarrow g g$. One of the challenges to generate detector-level events for the interference terms is that some of the generated events have negative cross sections. (Simply generating a full QCD $+X$ sample and then subtracting the pure QCD part is not practical, because of the very poor statistics. The actual LHC experiment has much better statistics than our simulations can provide.) In order to keep track of the events with positive and negative cross sections, the partonlevel events for the interference terms are divided into two sets: one with positive cross sections, and one with negative
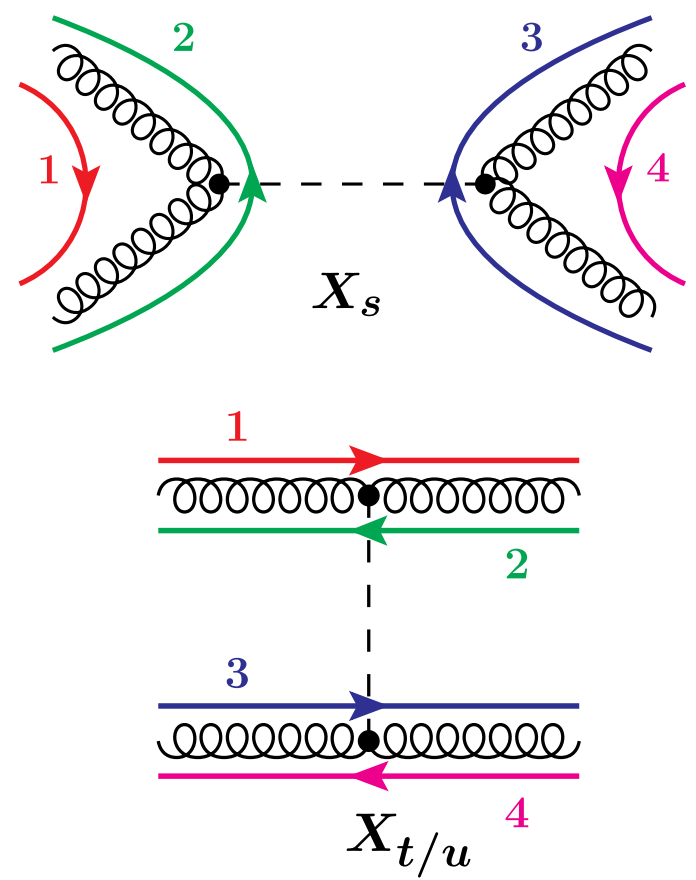

cross sections. The detector-level events for both sets are independently obtained. Then, to obtain the dijet invariant-mass distributions for the interference terms, the distributions with negative cross-section events are subtracted bin by bin from the ones with positive crosssection events.

Another problem is that MADGRAPH cannot assign a unique color flow [117-119] for each parton-level event for the interference between the QCD background $g g \rightarrow g g$ and color-singlet resonant signal $g g \rightarrow X \rightarrow g g$, thus preventing PYTHIA from performing showering and hadronization, thereby precluding the generation of any detector-level events. To get around this, we independently generated detector-level events for the interference between the QCD background and the color-singlet resonant signal by simply assigning a color flow by fiat to each event. There are four physically distinct color-flow possibilities, as shown in Fig. 8. We therefore repeated the analysis four times, each time with the same color flow assigned to every event. Thus, for color-singlet resonances, we obtain a spread of the possible differential cross sections by taking the maximum and minimum of the four possibilities in each invariant-mass bin, as shown in Figs. 10, 16, 18, and 24. On the other hand, this problem does not occur for color-octet resonances, as they are uniquely determined to have the same color charge as that of a gluon.

We generated at least $5 \times 10^{6}$ events for both the digluon resonant signal and the interference between the signal and
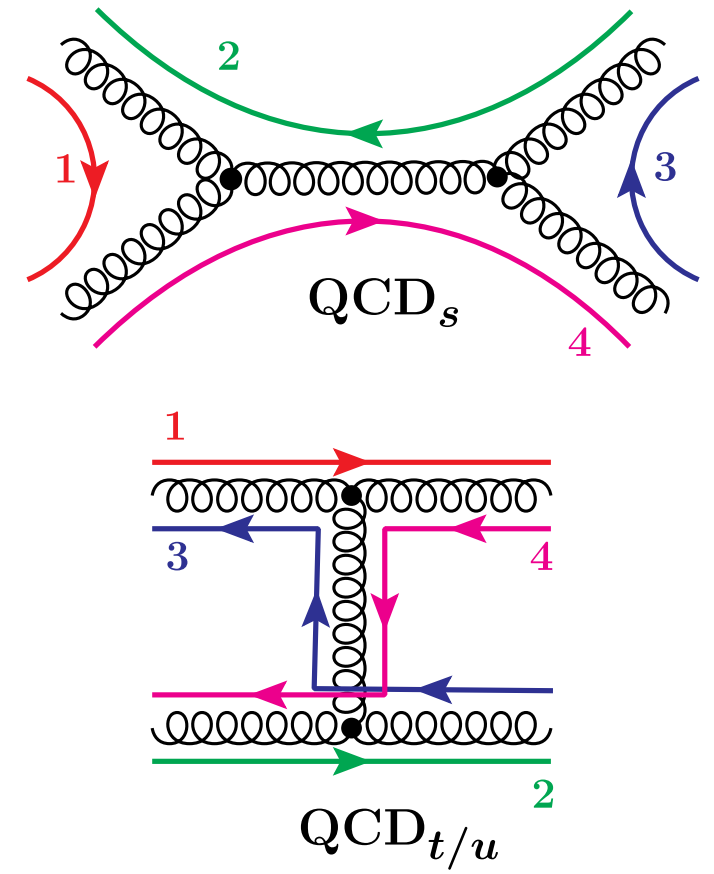

FIG. 8. The four distinct color flows for the parton-level events for the interference between the color-singlet digluon resonant process $g g \rightarrow X \rightarrow g g$ and the QCD process $g g \rightarrow g g$. These color flows are referred to in the text as $X_{s}, X_{t / u}, \mathrm{QCD}_{s}$, and $\mathrm{QCD}_{t / u}$, as labeled. The integer tags (e.g., 1-4) for the color flow lines are required by the showering and hadronization event generators to begin the parton shower. For example, in PYTHIA, the integer tags are typically assigned as 501-504. 
the QCD background, for $p p$ collisions at $\sqrt{s}=13 \mathrm{TeV}$, for each of the considered digluon resonant benchmark mass models. While generating events with MADGRAPH, we used MADGRAPH's default PDFs based on the NNPDF2.3 LO set [111] with factorization and renormalization scales $\mu_{F}=\mu_{R}=M_{X}$.

In our analysis, all of the jets are required to satisfy a cut on pseudorapidity $\left|\eta_{j}\right|<2.5$, as noted above in Eq. (3.53). To obtain the dijet mass $\left(m_{j j}\right)$, we follow the procedure used by CMS in Refs. [1,2] to reduce the sensitivity to radiation of additional gluons from the final-state gluons in the hardscattering event. Specifically, we start with the two leading $p_{T}$ jets, and the four-vectors of all other jets within $\Delta R=$ $\sqrt{(\Delta \eta)^{2}+(\Delta \phi)^{2}}<1.2$ of the two leading $p_{T}$ jets are added to the nearest leading jet to obtain two wide jets. These wide jets are then required to satisfy the cut $p_{T_{j}}>p_{T_{j}}^{\text {wide-jets }}=$ $100 \mathrm{GeV}$, as noted above in Eq. (3.52). Finally, as already mentioned in the previous section, we follow CMS by defining the signal region to have a cut on the difference in pseudorapidity for the two wide jets: $\left|\Delta \eta_{j j}\right|<1.3$ (for resonance masses smaller than $1.8 \mathrm{TeV}$ as in Ref. [1]) and $\left|\Delta \eta_{j j}\right|<1.1$ (for resonance masses larger than $1.8 \mathrm{TeV}$ as in Ref. [2]). This preferentially eliminates the pure background events, and tends to improve the efficiency for pure-resonance events compared to interference. However, as we will see it certainly does not reduce the effects of interference to a negligible level.

\section{RESULTS FOR $\Gamma=\Gamma_{g g}$}

In this section, we show results obtained for our benchmark model cases. In each case, we start with results obtained using the simple method of parton-level generation with smearing as outlined in Sec. III A. These are followed by results for the full detector simulation of Sec. III B. In this section, we consider the case $\Gamma_{g g}=\Gamma$, for which the relative effects of the interference are minimized.

\section{A. Spin 0, color singlet}

\section{Parton level with smearing}

The left panels of Fig. 9 show the leading-order digluon invariant-mass distributions, obtained by parton-level leading-order calculation, for $p p$ collisions at $\sqrt{s}=13 \mathrm{TeV}$, for a spin-0 color-singlet resonance, for benchmark examples from Table I with resonance masses of 1000, 2000, and $3000 \mathrm{GeV}$ in the top, middle, and bottom rows, respectively. We recall that the chosen benchmark values of $\Gamma_{g g}=\Gamma$ predict a resonant-only cross section about equal to the current CMS limit from Ref. [2]. The parton-level distributions are in the left column, and the right column shows the results after smearing by convolution with approximate detector responses (as shown in Fig. 7), to obtain a rough estimate of dijet mass distributions. In all six panels, the red lines show the naive results for the resonant signal $g g \rightarrow X \rightarrow g g$ with all $X$ exchange diagrams, while the blue lines show the full results including the interferences with the QCD background $g g \rightarrow g g$. (The pure QCD background contribution is much larger and is not shown, here or in the following.)

From Fig. 9, we see that when interferences with QCD amplitudes are included, the dijet mass distributions (blue lines) are both qualitatively and quantitatively different from that of distributions with only the $X$ exchange diagrams (red lines). In the parton-level results in the left column panels, with interference included, we see a peak below and a dip above the resonance mass rather than just a resonance peak. The origin of the peak/dip signature can be traced back to the term $\hat{s}-M_{X}^{2}$ in $d \hat{\sigma}_{s, \mathrm{QCD}} / d z$, as given in Eq. (3.13). The magnitude of the interference is, in general, enhanced for $\sqrt{\hat{s}}$ below $M_{X}$ because of the steeply falling gluon PDFs. After smearing (right column panels), the dip at higher invariant masses manifests as a deficit of events compared to the naive resonance results. For the results with interference included, we can also note that the peak below the resonance mass is significantly larger than the naive resonance peak that we get from not including the interference terms. However, the larger peak is counteracted by the fact that it is connected to a large low-energy tail. If the low-energy tail is absorbed into a QCD background fit (which we do not attempt in this paper, and can probably be done in a variety of distinct ways), an effective dip for $m_{j j}>M_{X}$ would probably result. We have checked that the interference effect would have been larger without the imposition of the $\Delta \eta$ cut. Correctly deriving a limit on these distribution shapes as new physics sources might require a more flexible analysis strategy than just modeling a resonance peak, even if the pure-resonance contribution is very narrow compared to the experimental resolution.

As the experimental data sets will grow in the future, allowing one to probe models with even smaller cross sections, we have checked that the relative importance of the interference and the pure resonance at a given $M_{X}$ stays nearly constant. We have also checked that this feature holds true for the other digluon resonances considered in this paper. On the other hand, as the mass is increased, Fig. 9 shows that the relative importance of the interference tends to increase substantially, particularly in the lowmass tail.

\section{Monte Carlo results with showering, hadronization, and detector simulation}

In Fig. 10, we show the dijet invariant-mass distributions for the considered benchmarks of Table I with $\Gamma=\Gamma_{g g}$, at the $13 \mathrm{TeV}$ LHC, for spin- 0 color-singlet resonances, this time obtained using Monte Carlo generation of events followed by simulations with showering, hadronization and 

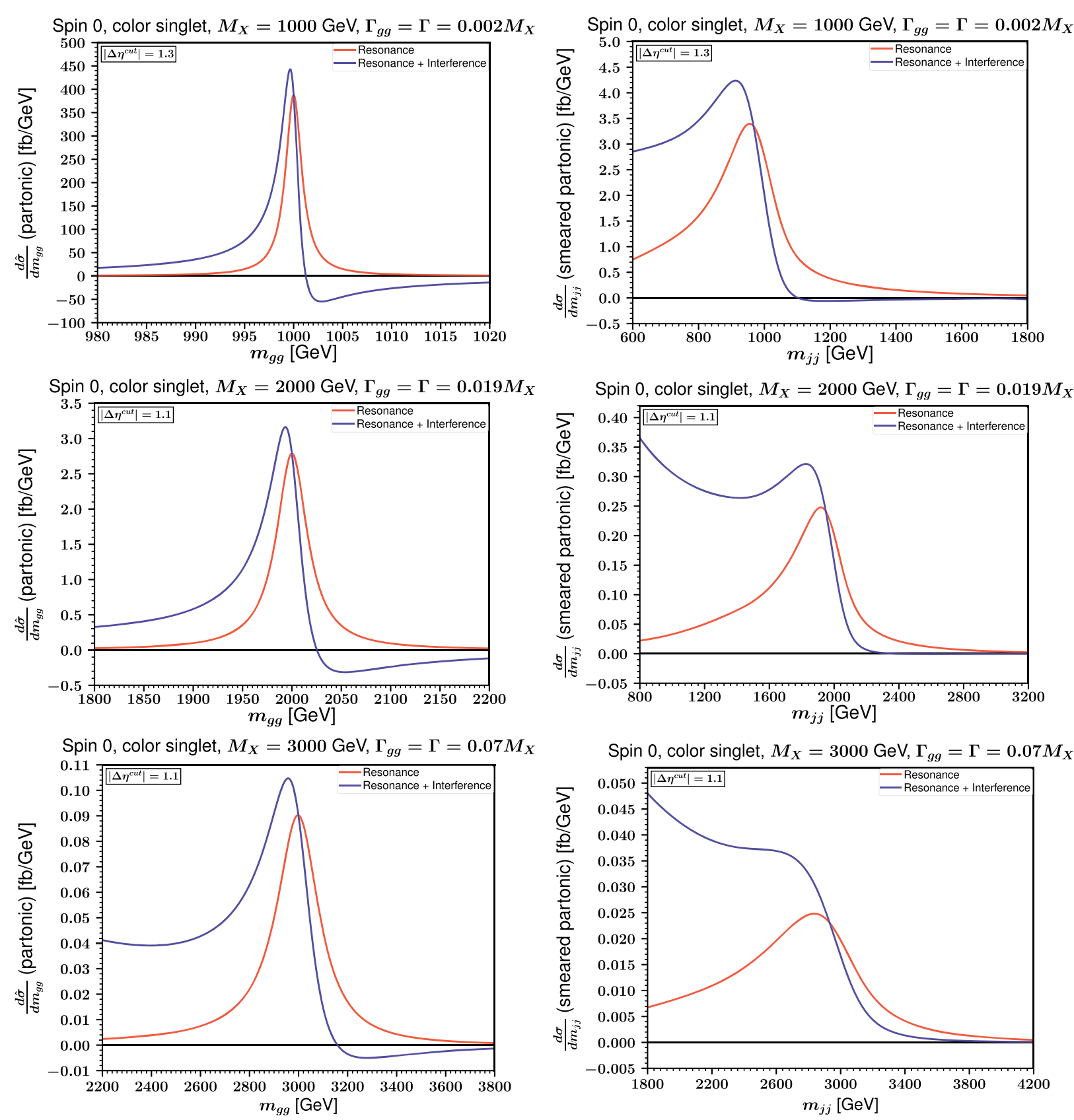

FIG. 9. Digluon invariant-mass distributions, at the $13 \mathrm{TeV}$ LHC, for spin-0 color-singlet resonances with $\left(M_{X}, \Gamma / M_{X}\right)=$ (1000 GeV, 0.002) (top row), (2000 GeV, 0.019) (middle row), and (3000 GeV, 0.07) (bottom row). The parton-level distributions are shown in the left column panels. These are then smeared by convolution with the estimated detector responses shown in Fig. 7 to obtain the dijet invariant-mass distributions in the right column panels. In all six panels, the red lines show the naive results for the resonant signal $g g \rightarrow X \rightarrow g g$, while the blue lines show the full results including the interferences with the QCD background $g g \rightarrow g g$.

detector simulation. $^{2}$ The results are shown for a digluon resonant process $g g \rightarrow X \rightarrow g g$, which include only the

\footnotetext{
${ }^{2}$ As a check of our implementation of the $X$ interactions using FeynRules, we verified that the parton-level distributions obtained using MADGRAPH (not shown here) closely match the parton-level distributions obtained by our calculations as described in Sec. III A and shown in Fig. 9. A similar check was done for each of the other (spin, color) combinations for $X$.
}

$s^{-}, t$-, and $u$-channel exchanges of $X$ (red line), and the full results. Here, the shaded blue region is the envelope of the results for the four possible color flows, as discussed above in reference to Fig. 8.

From Fig. 10, we note that the impact of QCD interference is smallest if we assume that the color flow is always the one that we have labeled $X_{s}$ (corresponding to the $s$-channel resonant process), and is greatest if we instead assume either the $X_{t / u}$ and $\mathrm{QCD}_{t / u}$ color flows, 

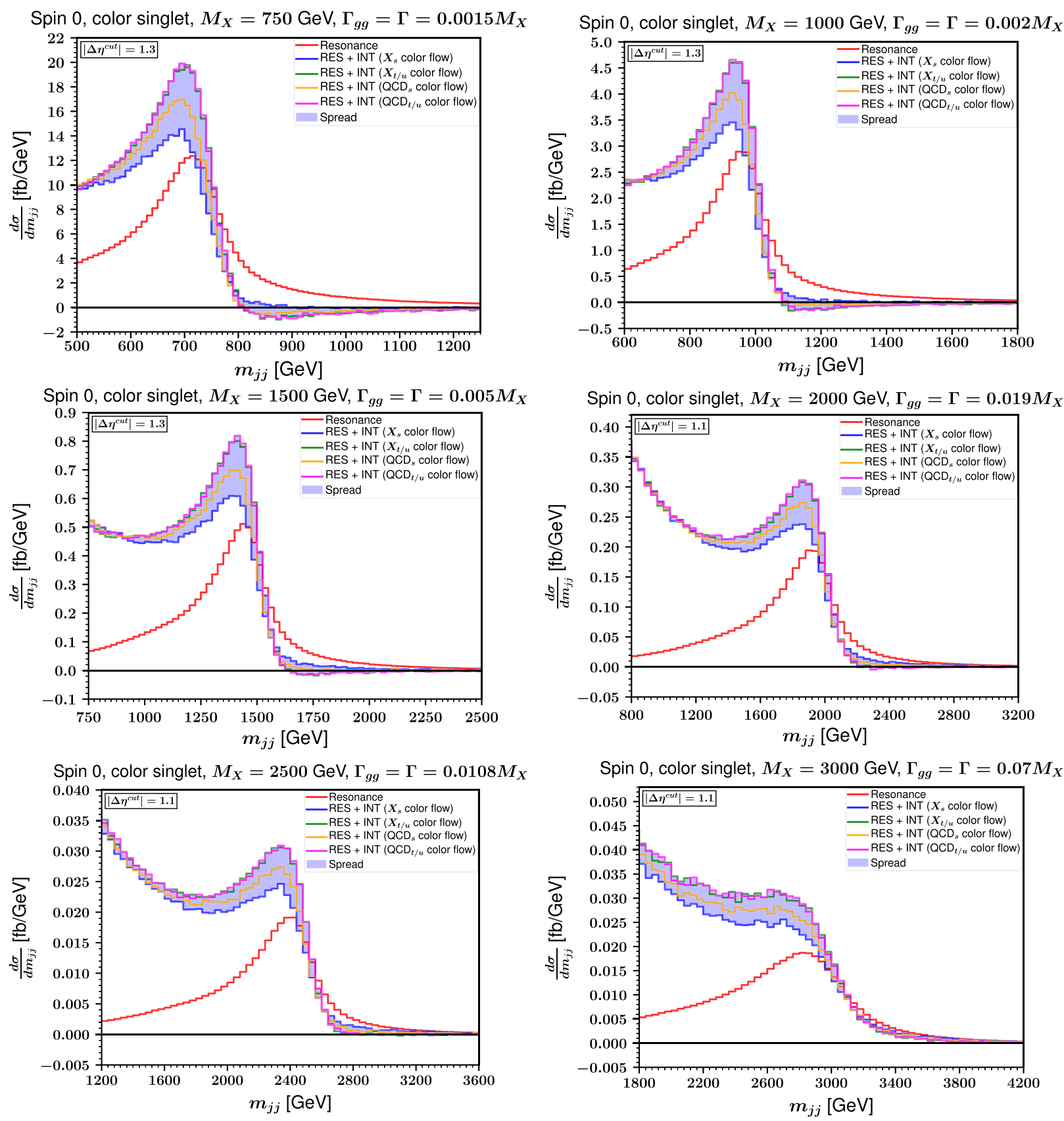

FIG. 10. Dijet invariant-mass distributions for the spin-0 color-singlet benchmarks of Table I with $\Gamma=\Gamma_{g g}$, at the $13 \mathrm{TeV} \mathrm{LHC}$, obtained with showering, hadronization, and detector simulation. The red lines show the naive results with only the resonance diagrams of the $g g \rightarrow X \rightarrow g g$ process (RES), which include the $s$-, $t$-, and $u$-channel exchanges of $X$, while the other four colored lines show the full results including interferences with the continuum QCD $g g \rightarrow g g$ amplitudes (INT) for all four color flows shown in the previous Fig. 8, as labeled. The shaded region shows the spread in the full result in each invariant-mass bin for different color flow choices. The cases shown in the right column can be compared directly to those in the right column of the previous Fig. 9 based on the more simplistic method of smearing parton level distributions by convolution with an approximate detector response dijet mass distribution.

which produce almost identical results. The color flow labeled $\mathrm{QCD}_{s}$ produces intermediate results. In any case, the QCD interference again is seen to change the naively expected resonance peak shape in a way consistent with the previous discussion. Again, we note that the relative importance of interference seems to increase with the resonance mass. The right-hand panels of Fig. 10 can be compared to the right-hand panels of Fig. 9, as both have the same masses $M_{X}=1000,2000,3000 \mathrm{GeV}$. The match is of course not an exact one, because the smearing method using the detector responses in Fig. 7 is only a very rough approximation to the full-fledged event generation with 
showering, hadronization, and detector simulation, and furthermore the color-flow uncertainty is evidently a nontrivial one. The more complete treatment tends to give lower yields in this case. However, the qualitative similarity between the results of the two methods is a useful check. Even the more complete detector simulation is of course different from the true CMS and ATLAS detector responses.

\section{B. Spin 0, color octet}

\section{Parton level with smearing}

In Fig. 11 we show the parton-level digluon invariant-mass distributions for $p p$ collisions at $\sqrt{s}=13 \mathrm{TeV}$, for massive spin-0 color-octet resonances, for the benchmark examples of Table I with masses 1000, 2000, and $3000 \mathrm{GeV}$ in the top,
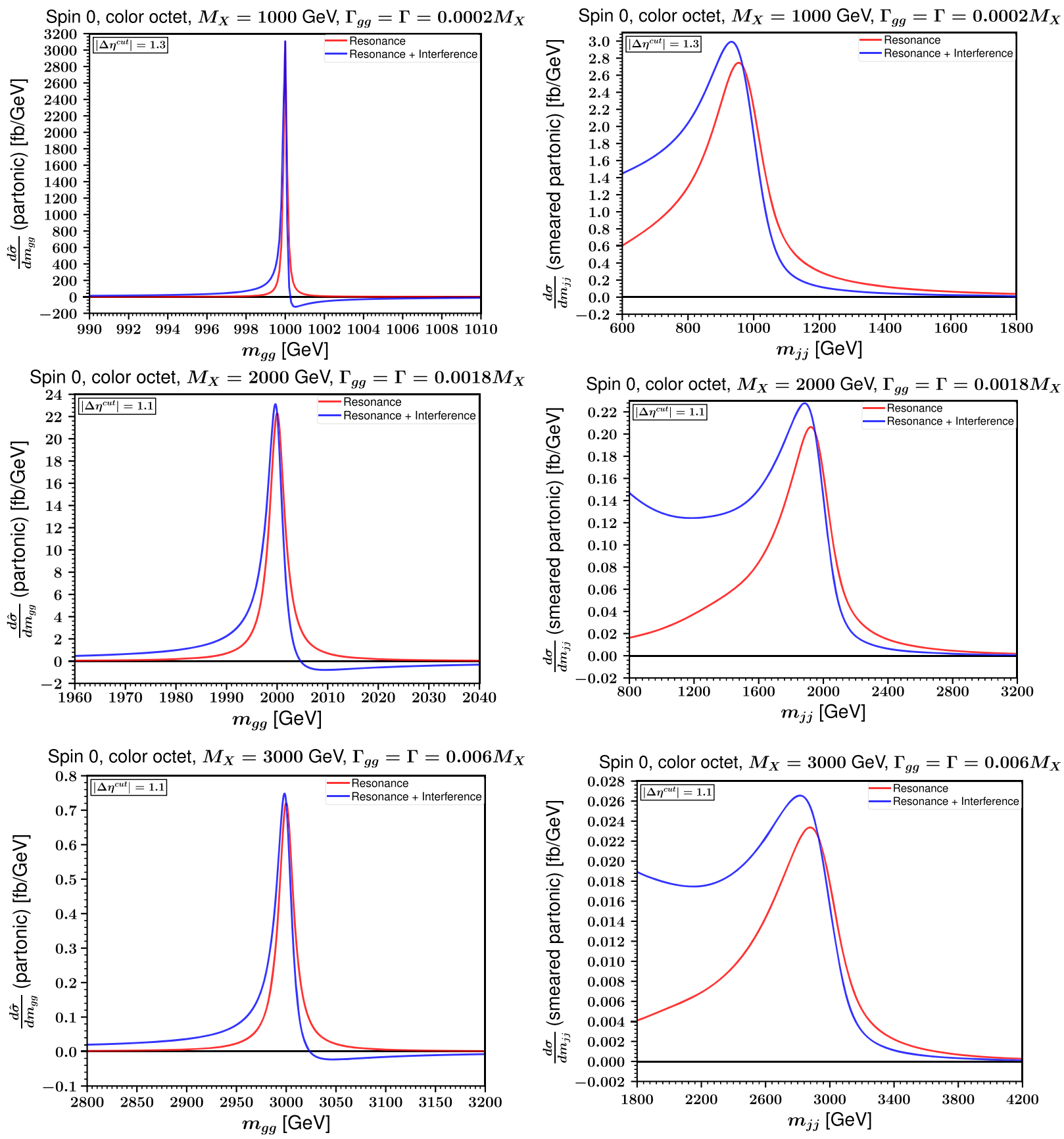

FIG. 11. Digluon invariant-mass distributions, at the $13 \mathrm{TeV} \mathrm{LHC}$, for spin-0 color-octet resonances with $\left(M_{X}, \Gamma / M_{X}\right)=$ (1000 GeV, 0.0002) (top row), (2000 GeV, 0.0018) (middle row), and (3000 GeV, 0.006) (bottom row). The parton-level distributions are shown in the left column panels. These are then smeared by convolution with the detector responses shown in Fig. 7, to obtain the dijet invariant-mass distributions in the right panels. In all six panels, the red lines show the naive results for the resonant signal $g g \rightarrow X \rightarrow g g$, while the blue lines show the full results including the interferences with the QCD background $g g \rightarrow g g$. 
middle, and bottom rows, respectively. As in the previous subsection, the parton-level distributions before smearing are shown in the left panels, and the right panels show the distributions after convolution with approximate detector responses shown in Fig. 7, as described in Sec. III A. In all six panels, the red lines show the results for the resonant signal $g g \rightarrow X \rightarrow g g$ with all $X$ exchange diagrams, while the blue lines show the full results including the interferences with the QCD amplitudes $g g \rightarrow g g$.

Just as was the case for spin- 0 color-singlet resonances, the parton-level results before smearing show a peak below $m_{j j}<M_{X}$ and a dip above $m_{j j}>M_{X}$ for the full result (blue line), as opposed to a pure peak (red line) that we get without including the interference terms. After smearing,
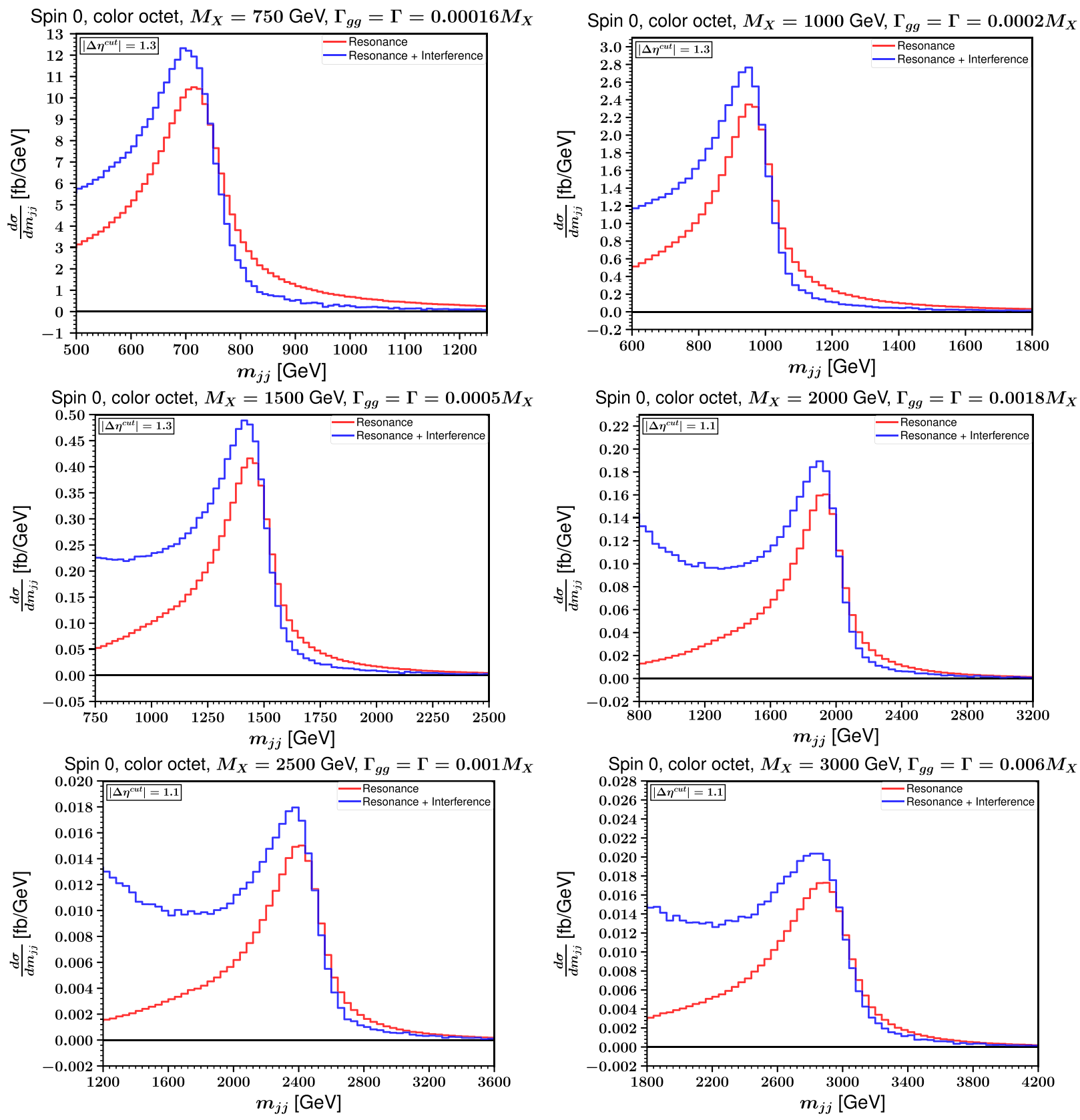

FIG. 12. Dijet invariant-mass distributions for the spin-0 color-octet benchmarks of Table I with $\Gamma=\Gamma_{g g}$, at the 13 TeV LHC, obtained with showering, hadronization, and detector simulation. The red lines show the naive results with the resonance diagrams of the $g g \rightarrow X \rightarrow g g$ process (RES), which include the $s^{-}, t$-, and $u$-channel exchanges of $X$, while the blue lines show the full results including interferences with the continuum QCD $g g \rightarrow g g$ amplitudes (INT). The cases shown in the right column can be compared directly to those in the right column of the previous Fig. 11 based on the more simplistic method of smearing parton level distributions by convolution with an approximate detector response dijet mass distribution. 
the deficit above the input resonance mass, compared to the naive result, does not appear to be as relevant as in the color-singlet case. The main feature is again the presence of the low-mass positive tail. Depending on how this would be absorbed into the QCD background fit, this could again lead to both a peak slightly below $M_{X}$ and an apparent dip in the differential distribution above $M_{X}$. However, the interference effects for spin-0 color-octet resonances are not as big as for spin-0 color singlets, as we anticipated above in the discussion immediately following Eq. (3.30).

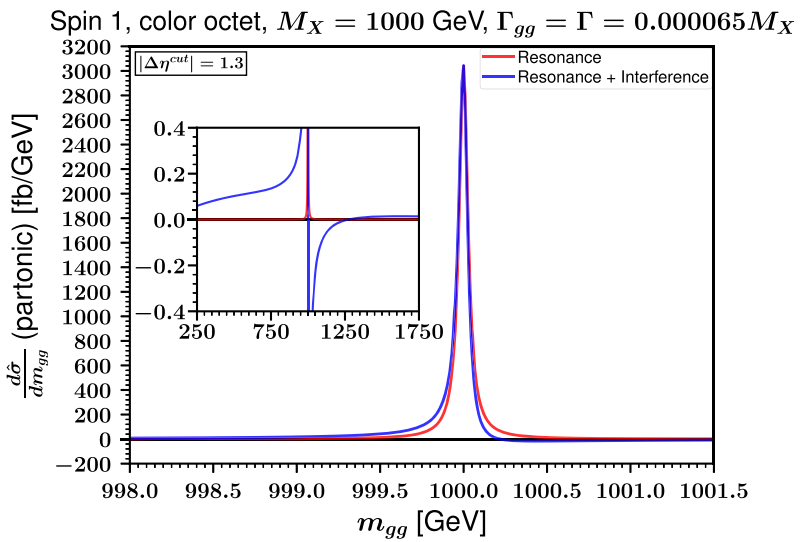

Spin 1, color octet, $M_{X}=2000 \mathrm{GeV}, \Gamma_{g g}=\Gamma=0.00054 M_{X}$
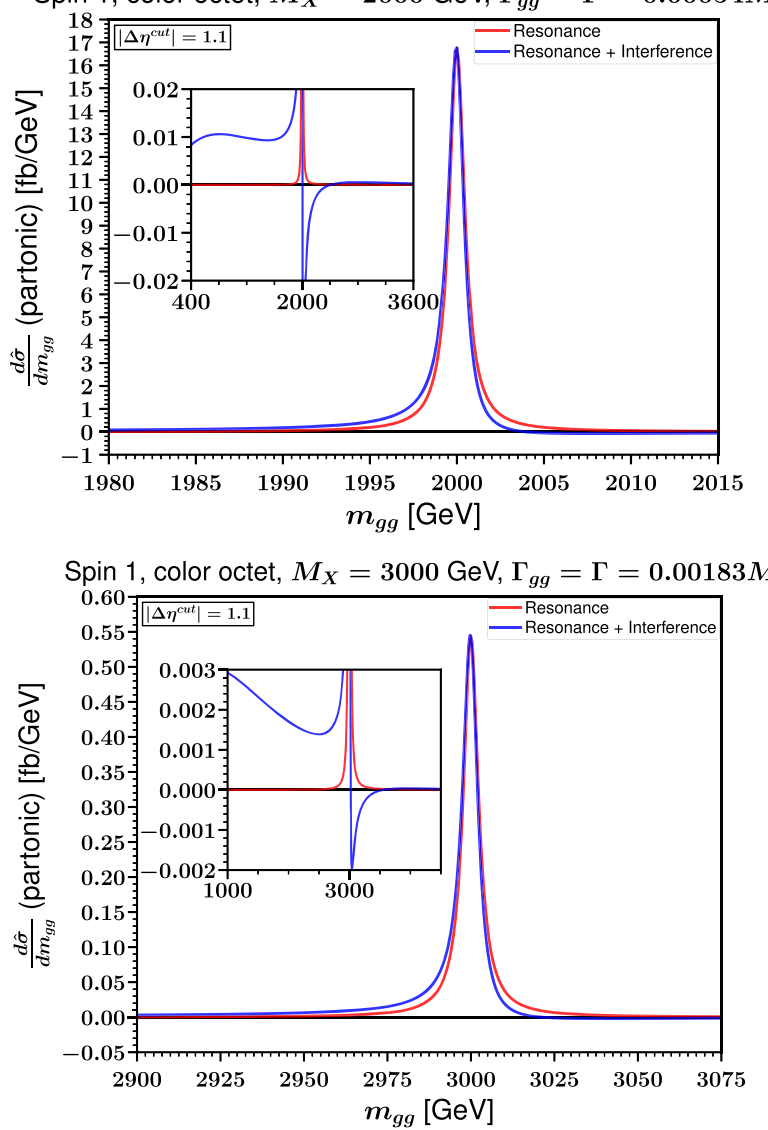

Spin 1, color octet, $M_{X}=1000 \mathrm{GeV}, \Gamma_{g g}=\Gamma=0.000065 M_{X}$
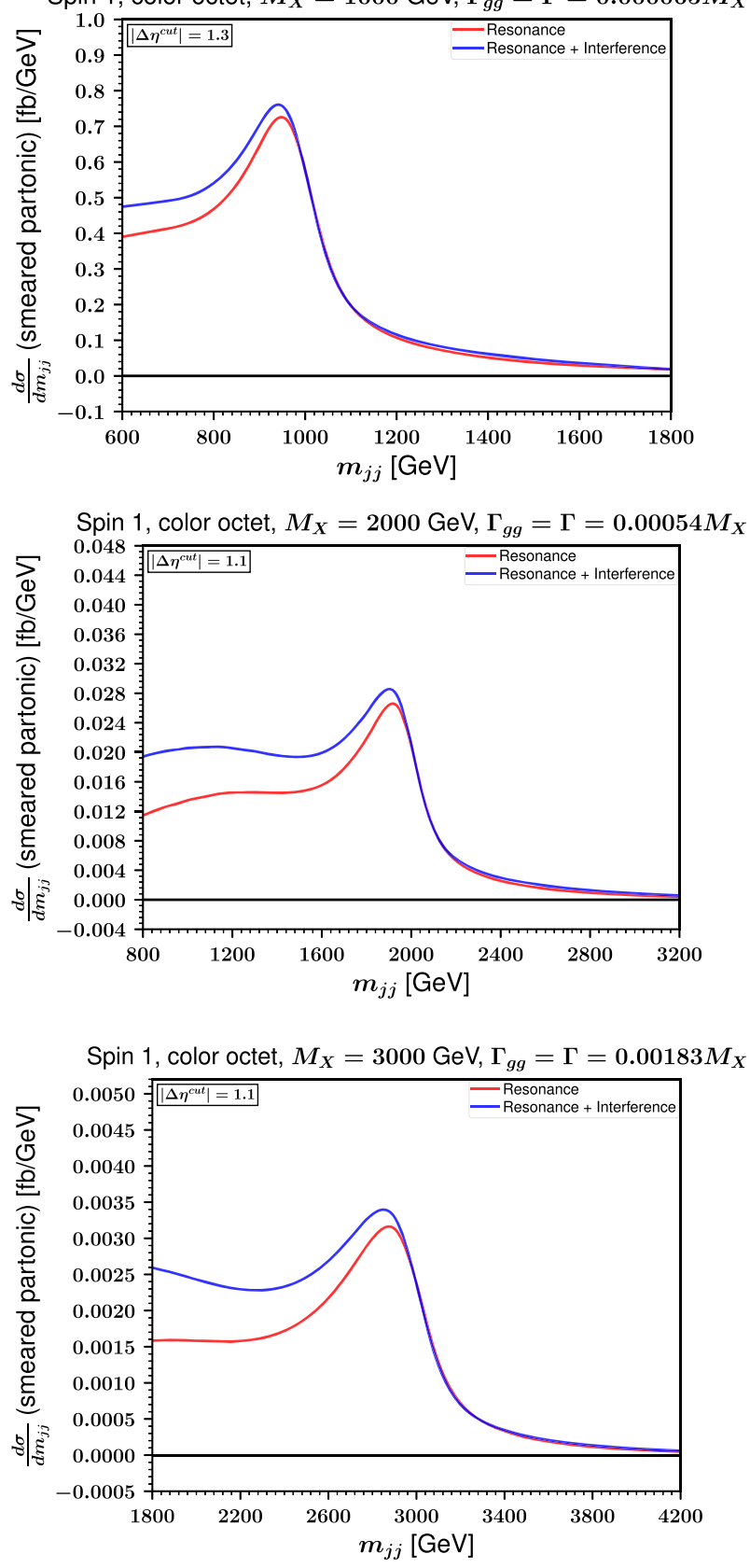

FIG. 13. Digluon invariant-mass distributions, at the $13 \mathrm{TeV} \mathrm{LHC}$, for spin-1 color-octet resonances with $\left(M_{X}, \Gamma / M_{X}\right)=$ $\left(1000 \mathrm{GeV}, 6.5 \times 10^{-5}\right)$ (top row), $\left(2000 \mathrm{GeV}, 5.4 \times 10^{-4}\right)$ (middle row), and (3000 GeV, 0.00183) (bottom row). The parton-level distributions (left panels) are smeared by convolution with the detector response, shown in Fig. 7, to obtain the dijet invariant-mass distributions (right panels). In all six panels, the red lines show the naive results for the resonant signal $g g \rightarrow X \rightarrow g g$, while the blue lines show the full results including the interferences with the QCD background $g g \rightarrow g g$. The inset plots within the left panels and their enclosing plots show the same data but with different scales on the axes. 


\section{Monte Carlo results with showering, hadronization, and detector simulation}

Figure 12 shows the dijet invariant-mass distributions for the considered benchmarks of Table I where $X$ is assumed to always decay to a pair of gluons, at the $13 \mathrm{TeV}$ LHC, for spin- 0 color-octet resonances, obtained using Monte Carlo simulations. The results are shown for the naive resonant process $g g \rightarrow X \rightarrow g g$ and for the full results, which also include the interferences with the continuum QCD $g g \rightarrow g g$ amplitudes.

From Fig. 12, the QCD interference with a spin-0 coloroctet resonance has the aforementioned feature of having less dramatic positive tails in the region $m_{j j}<M_{X}$ with almost no negative tails in the region $m_{j j}>M_{X}$. Unlike the case of a color-singlet scalar, here we have exactly one
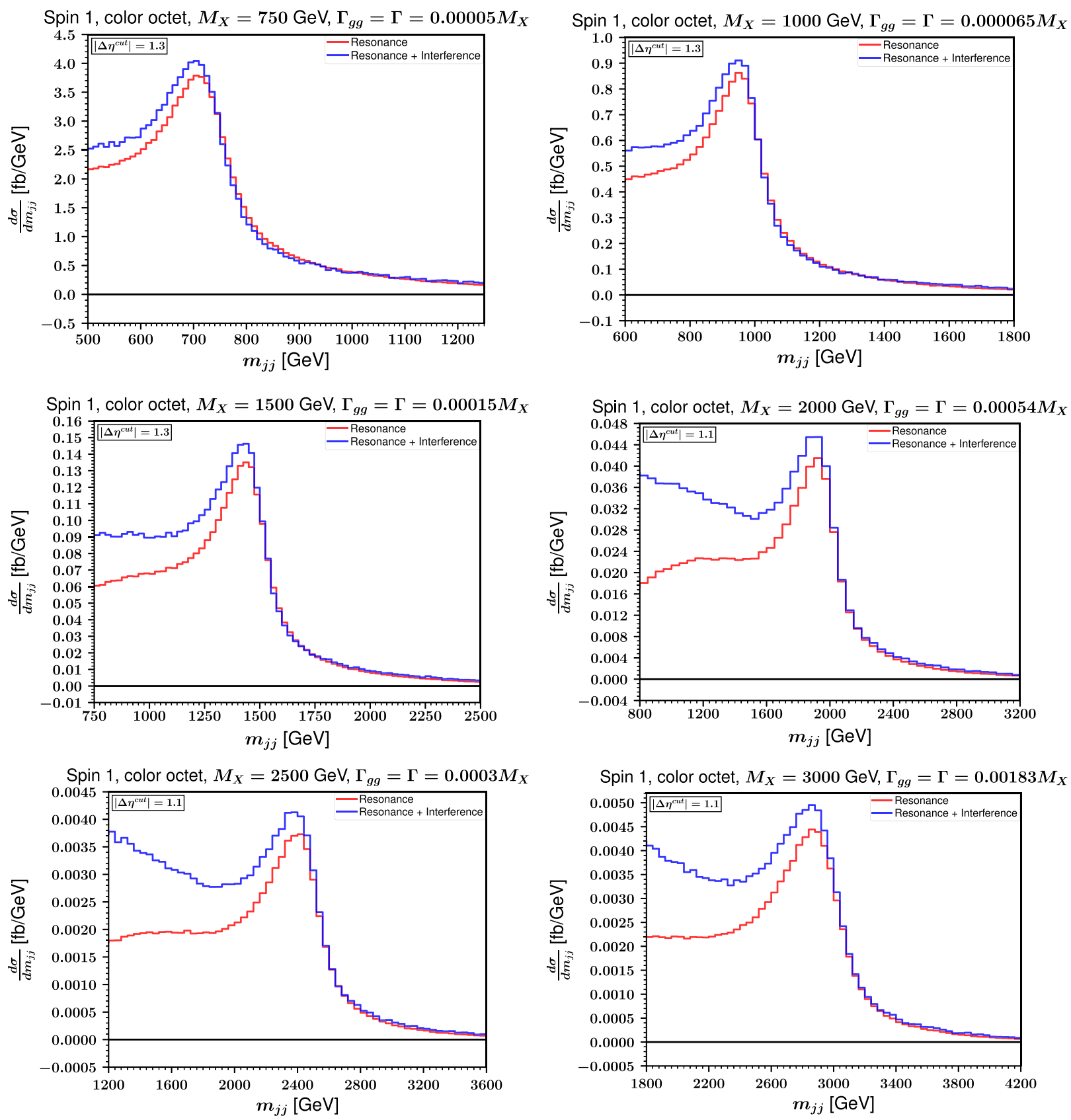

FIG. 14. Dijet invariant-mass distributions for the spin-1 color-octet benchmarks of Table I with $\Gamma=\Gamma_{g g}$, at the $13 \mathrm{TeV} \mathrm{LHC}$, obtained with showering, hadronization, and detector simulation. The red lines show the naive results with the resonance diagrams of the $g g \rightarrow X \rightarrow g g$ process (RES), which include the $s$-, $t$-, and $u$-channel exchanges of $X$, while the blue lines show the full results including interferences with the continuum QCD $g g \rightarrow g g$ amplitudes (INT). The cases shown in the right column can be compared directly to those in the right column of the previous Fig. 13 based on the more simplistic method of parton level with smearing. 
result for the QCD interferences because the color flow is uniquely determined. The relative importance of the interference again increases as one moves to higher resonance masses $M_{X}$. Again, comparing the right column panels of Fig. 12 to the corresponding right column panels of Fig. 11, which have the same masses (1000, 2000, and $3000 \mathrm{GeV}$ ), the fully simulated results do not exactly match with the smeared parton-level results, with the more complete simulation producing a lower yield, but the shapes are reassuringly qualitatively similar.
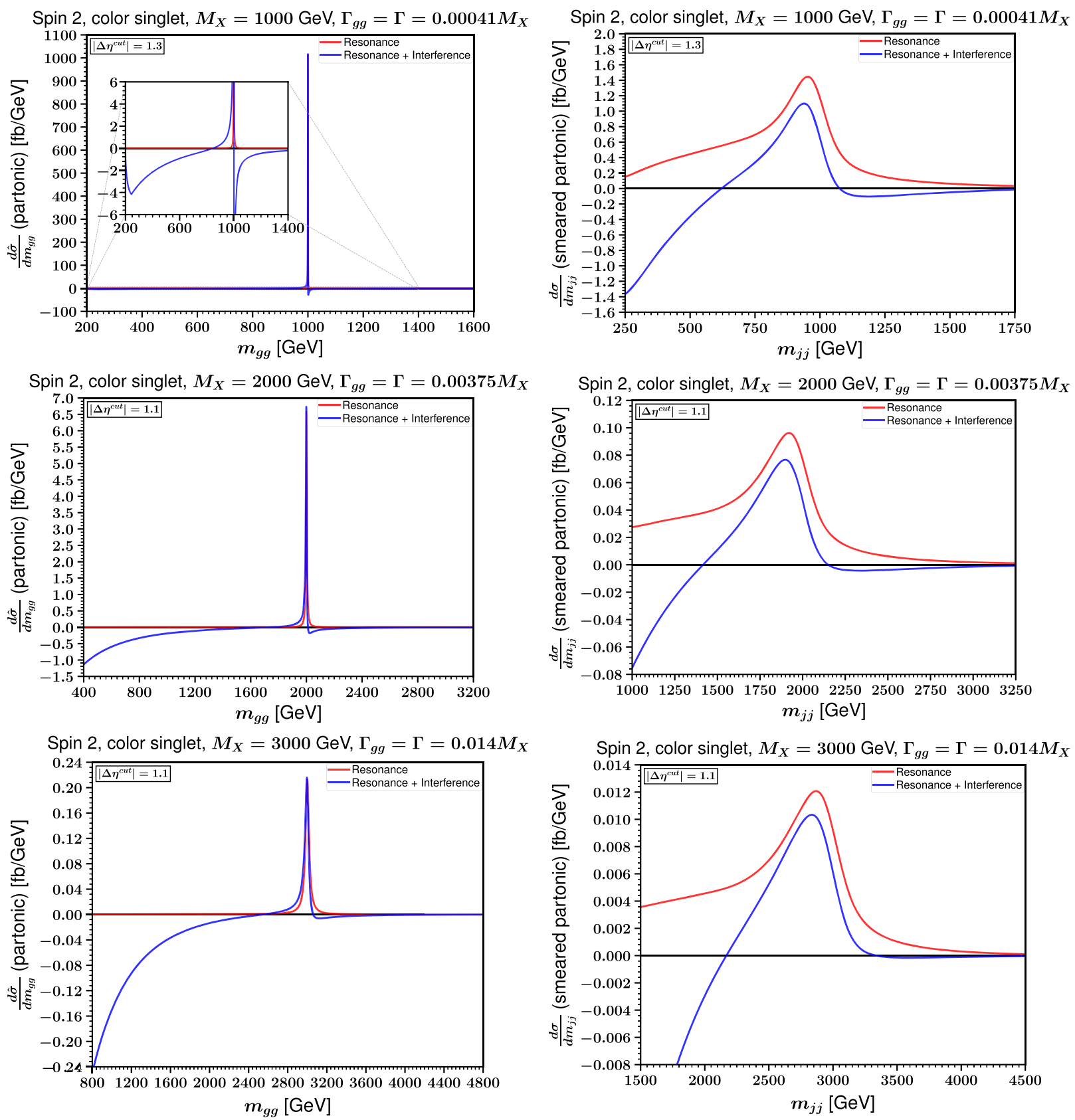

FIG. 15. Digluon invariant-mass distributions, at the $13 \mathrm{TeV} \mathrm{LHC}$, for spin-2 color-singlet resonances with $\left(M_{X}, \Gamma / M_{X}\right)=$ (1000 GeV,0.00041) (top row), (2000 GeV, 0.00375) (middle row), and (3000 GeV, 0.014) (bottom row). The parton-level distributions (left panels) are smeared by convolution with the detector response, shown in Fig. 7, to obtain the dijet invariant-mass distributions (right panels). In all six panels, the red lines show the naive results for the resonant signal $g g \rightarrow X \rightarrow g g$, while the blue lines show the full results including the interferences with the QCD background $g g \rightarrow g g$. The inset plot within the top-left panel shows the same data as its enclosing plot but with different scales on the axes. The negative tails at small invariant mass come from the interference between the QCD amplitudes and the $t$ - and $u$-channel $X$ exchange diagrams. 


\section{Spin 1, color octet}

\section{Parton level with smearing}

Figure 13 shows the digluon invariant-mass distributions, for $p p$ collisions at $\sqrt{s}=13 \mathrm{TeV}$, for spin-1 color-octet resonances with benchmark examples of Table I, namely, $\left(M_{X}, \Gamma / M_{X}\right)=\left(1000 \mathrm{GeV}, 6.5 \times 10^{-5}\right)$ (top row), $\left(2000 \mathrm{GeV}, 5.4 \times 10^{-4}\right)$ (middle row), and $(3000 \mathrm{GeV}$, 0.00183) (bottom row). As before, the parton-level results before smearing are shown in the left panels, and the
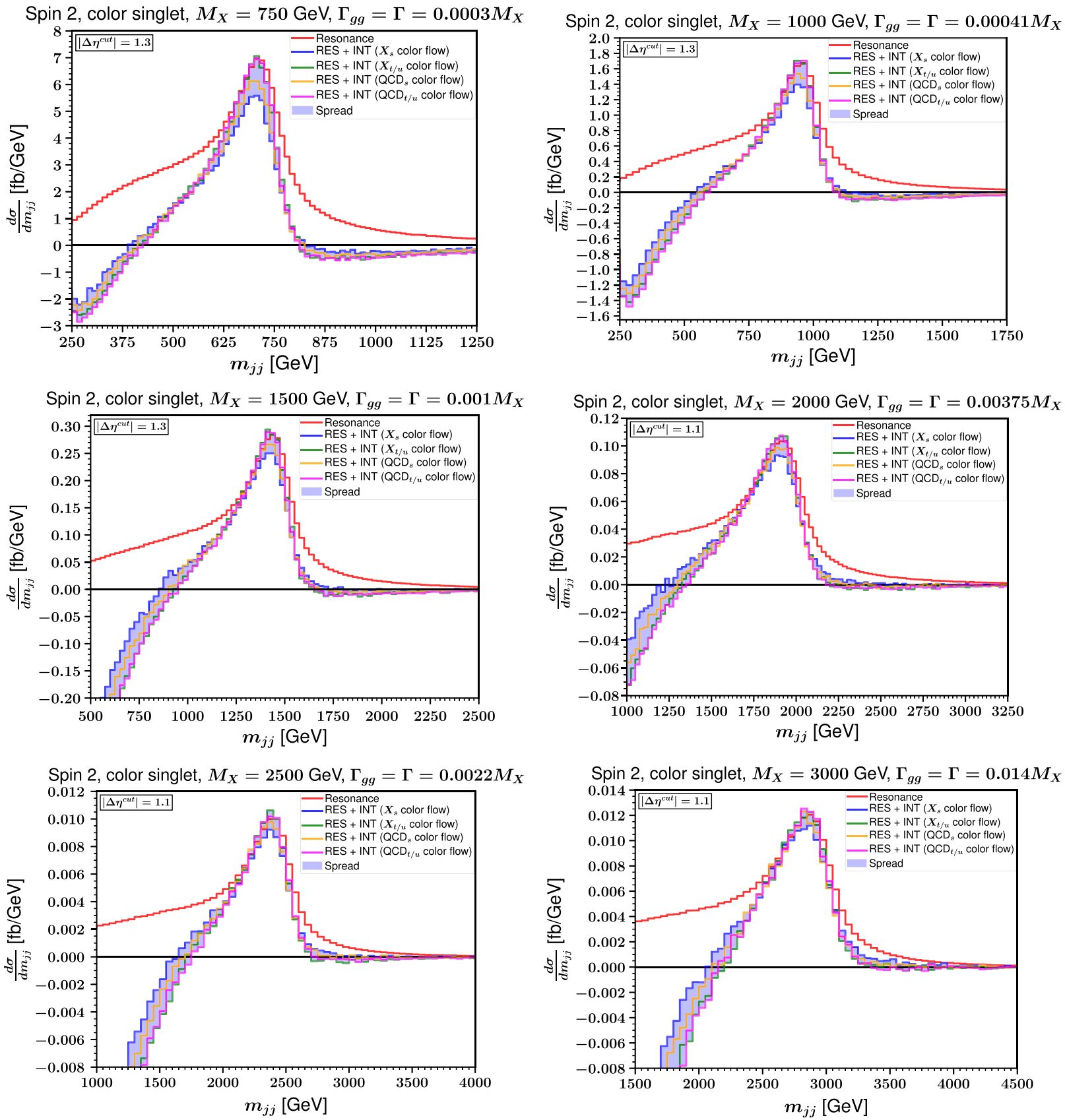

FIG. 16. Dijet invariant-mass distributions for the spin-2 color-singlet resonance benchmarks of Table $\mathrm{I}$ with $\Gamma=\Gamma_{g g}$, at the $13 \mathrm{TeV}$ LHC, obtained with showering, hadronization, and detector simulation. The red lines show the naive results with the resonance diagrams of the $g g \rightarrow X \rightarrow g g$ process (RES), which include the $s-, t$, and $u$-channel exchanges of $X$, while the other four colored lines show the full results including interferences with the continuum QCD $g g \rightarrow g g$ amplitudes (INT) for all four color flows shown in Fig. 8, as labeled. The shaded region shows the spread in the full result in each invariant-mass bin, for the four different color flow choices. The negative tails at small invariant mass come from the interference between the QCD amplitudes and the $t$ - and $u$-channel $X$ exchange diagrams. The cases shown in the right column can be compared directly to those in the right column of the previous Fig. 15 based on the more simplistic method of smearing parton level distributions by convolution with an approximate detector response dijet mass distribution. 
corresponding mass distributions after smearing are shown in the right panels. In all panels, the red lines show the results for the resonant signal $g g \rightarrow X \rightarrow g g$ with all $s, t, u$-channel $X$ exchange diagrams, while the blue lines show the full results including the interferences with the QCD background $g g \rightarrow g g$. In this case, the relative effects of the interference are seen to be of a similar character, but smaller than, the spin- 0 cases discussed above.

\section{Monte Carlo results with showering, hadronization, and detector simulation}

Figure 14 shows all of the dijet invariant-mass distributions for the considered benchmarks of Table I with $\Gamma=\Gamma_{g g}$, at the $13 \mathrm{TeV} \mathrm{LHC}$, for spin-1 color-octet resonances, obtained using Monte Carlo simulations. The results are shown for a digluon resonant process $g g \rightarrow X \rightarrow g g$, and the full results, which also include the interferences with the continuum QCD $g g \rightarrow g g$ amplitudes.

From Fig. 14 we see that the QCD interferences with spin-1 color-octet resonances (blue lines) have a peak below the resonance mass, which is almost comparable to the pure peak, obtained by excluding the interference terms. The differential cross sections in the region $m_{j j}>M_{X}$ are almost unaffected by including the interference. However, as before, the presence of the large lowmass tail means that after fitting the QCD background, the residual distribution may have an apparent deficit of events above $M_{X}$. Comparing the right columns of Figs. 13 and 14, we note that in this case the difference between the shapes found with the parton-level smearing method and the full event simulation method is more significant than in the spin- 0 case, this time with a larger yield and a more pronounced low-mass tail using the latter (presumably more accurate) method. As was the case with the colorsinglet resonances, the QCD interference seems to have a relatively larger impact at higher resonance masses than at smaller $M_{X}$.

\section{Spin 2, color singlet}

\section{Parton level with smearing}

We now turn to the case of a massive spin-2 color-singlet digluon resonance. In Fig. 15, we show the parton-level digluon invariant-mass distributions for $p p$ collisions at
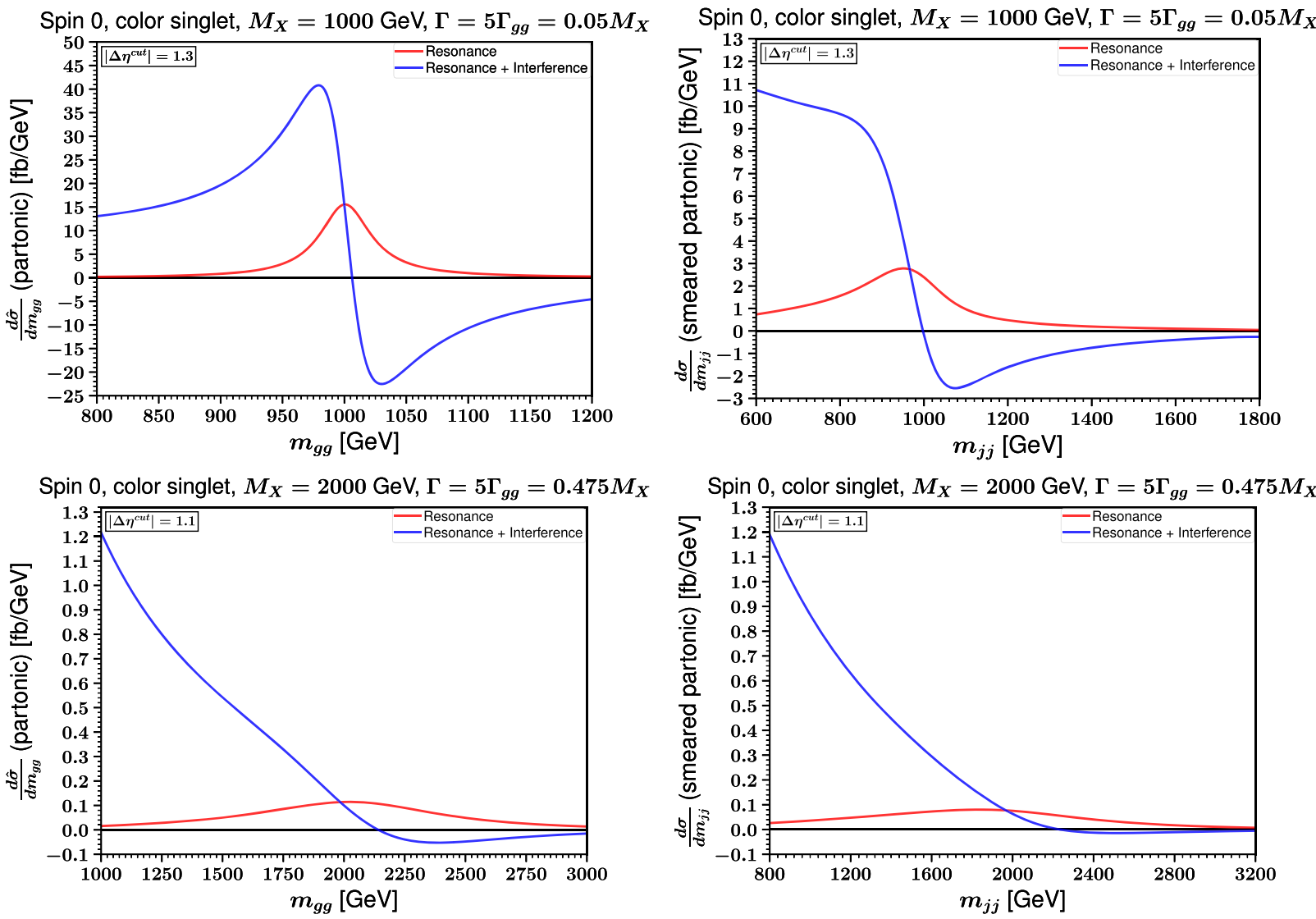

FIG. 17. Digluon invariant-mass distributions, at the $13 \mathrm{TeV} \mathrm{LHC}$, for benchmark spin-0 color-singlet resonances from Table I, with $\Gamma_{g g}=\Gamma / 5$, and $M_{X}=1000 \mathrm{GeV}$ (top row) and $2000 \mathrm{GeV}$ (bottom row). The parton-level distributions are shown in the left column panels. These are then smeared by convolution with the estimated detector responses shown in Fig. 7 to obtain the dijet invariant-mass distributions in the right column panels. In all four panels, the red lines show the naive results for the resonant signal $g g \rightarrow X \rightarrow g g$, while the blue lines show the full results including the interferences with the QCD background $g g \rightarrow g g$. 
$\sqrt{s}=13 \mathrm{TeV}$, for $\left(M_{X}, \Gamma / M_{X}\right)=(1000 \mathrm{GeV}, 0.00041)$ (top row), (2000 GeV, 0.00375) (middle row), and (3000 GeV, 0.014) (bottom row). As before, the results before smearing are shown in the left panels, and mass distributions after smearing are shown in the right panels. In all panels, the red lines show the results for the resonant signal $g g \rightarrow X \rightarrow g g$ with all $X$ exchange diagrams, while the blue lines show the full results including the interferences with the QCD $g g \rightarrow g g$ amplitudes.

In the spin-2 case, we note that there is a unique feature not found in the previous cases: the effect of the interference is negative for all dijet invariant masses well below $M_{X}$ after
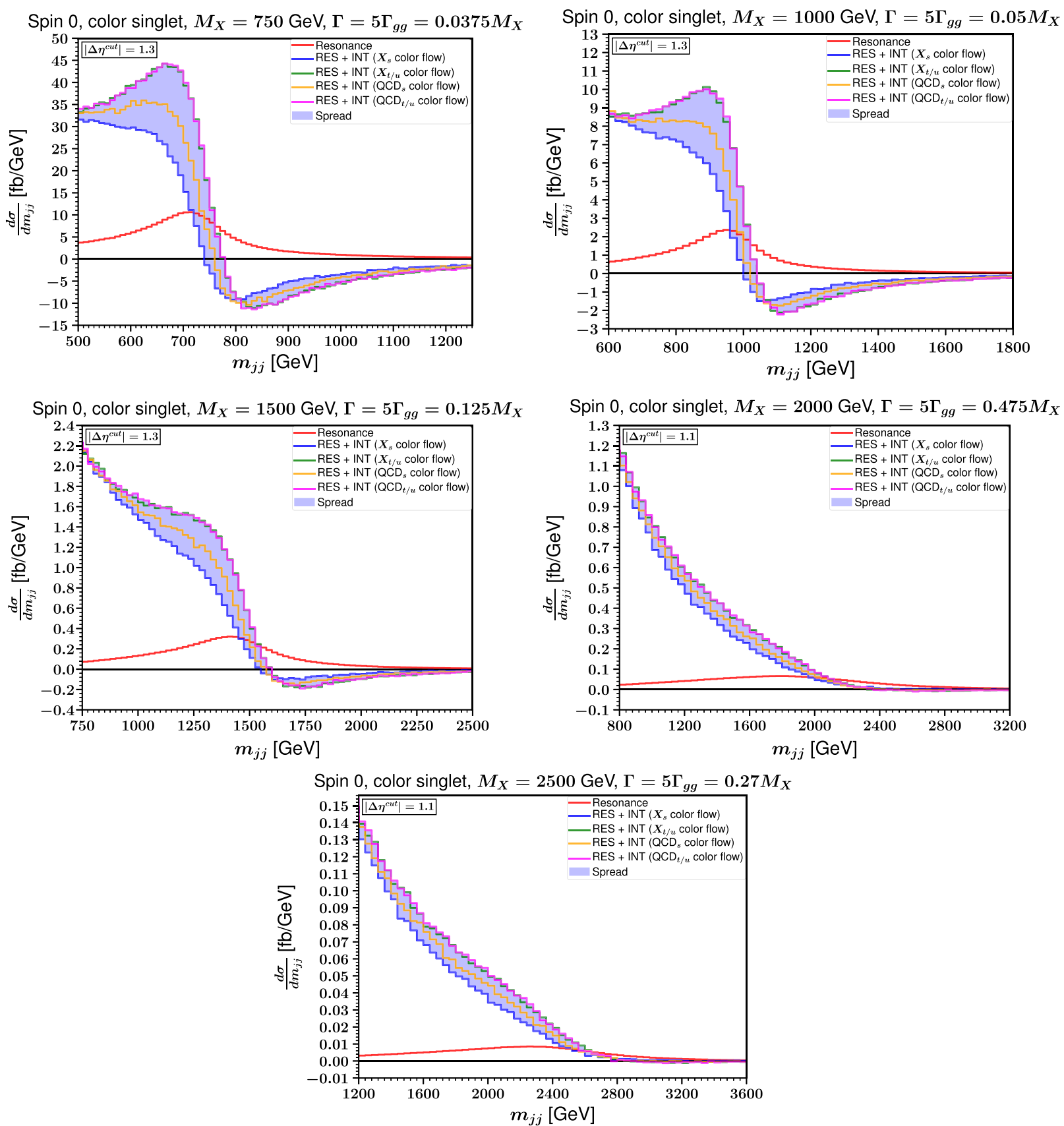

FIG. 18. Dijet invariant-mass distributions for the spin-0 color-singlet benchmarks of Table I with $\Gamma_{g g}=\Gamma / 5$, at the $13 \mathrm{TeV} \mathrm{LHC}$, obtained with showering, hadronization, and detector simulation. The red lines show the naive results with only the resonance diagrams of the $g g \rightarrow X \rightarrow g g$ process (RES), which include the $s$-, $t$-, and $u$-channel exchanges of $X$, while the other four lines show the full results including interferences with the continuum QCD $g g \rightarrow g g$ amplitudes (INT) for all four color flows shown in Fig. 8, as labeled. The shaded region shows the spread in the full result in each invariant-mass bin for the different color flow choices. The cases shown in the right column of the top and middle rows can be compared directly to those in the right column of the previous Fig. 17 based on the more simplistic method of smearing parton level distributions by convolution with an approximate detector response dijet mass distribution. 
smearing (but with a magnitude that of course varies with the mass). This can be traced in part to a large negative interference effect in the parton-level results for $m_{g g}$ well below $M_{X}$, due to the contributions from the interference between $t$ - and $u$-channel $X$ exchange diagrams and the QCD diagrams. Thus, at the parton level for spin-2 color-singlets, there is an interference dip in the regions $m_{g g} \ll M_{X}$ (although it is small compared to the large QCD background in that range of $m_{g g}$ ). This translates into a substantial negative low-mass tail compared to the naive pure-resonance result. There is then a steady rise until $m_{j j}$ is slightly less than $M_{X}$, followed by a dip in the regions $m_{j j}>M_{X}$. It would be interesting to see whether this pattern is maintained after including higher-order contributions.
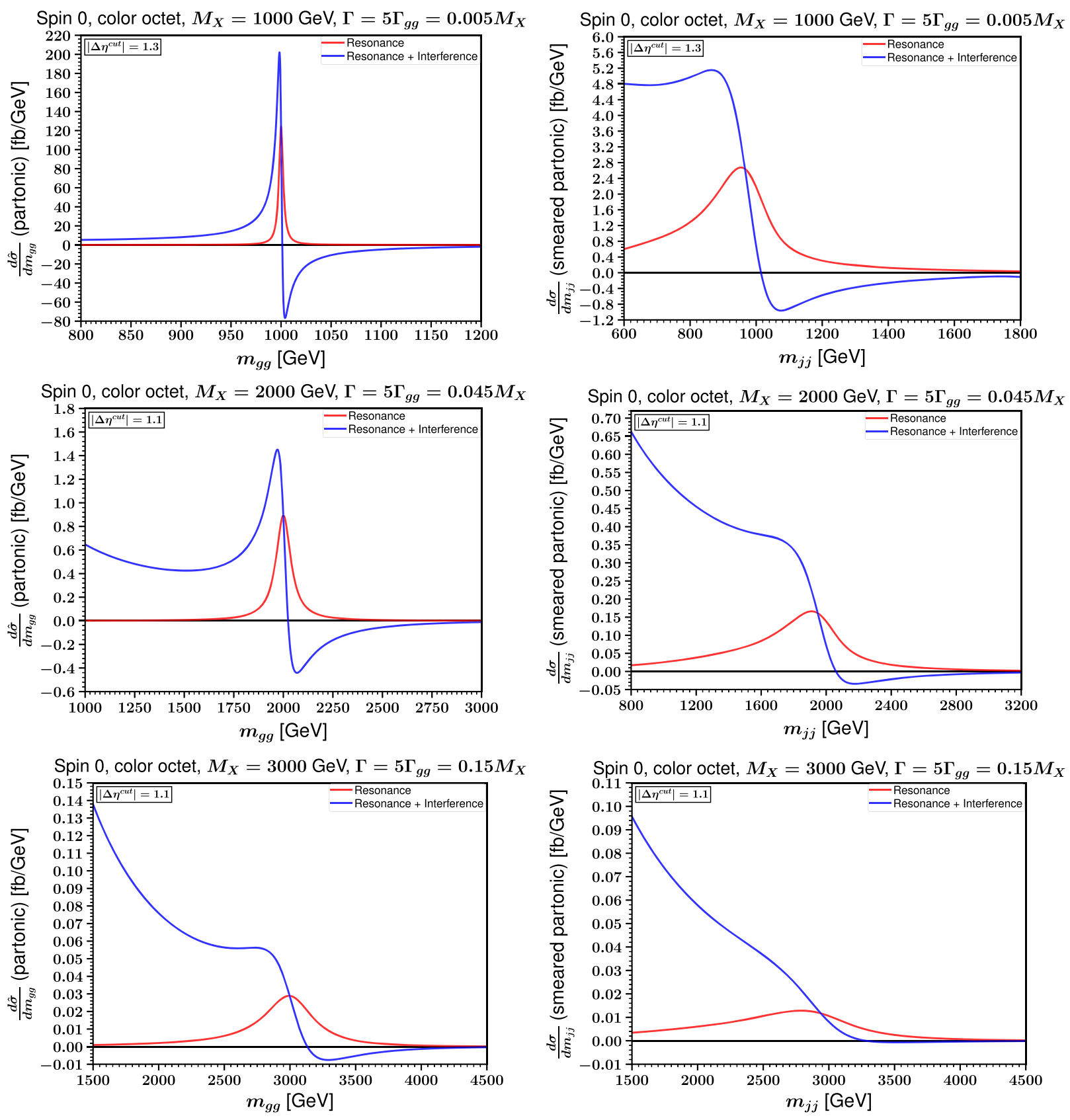

FIG. 19. Digluon invariant-mass distributions, at the $13 \mathrm{TeV} \mathrm{LHC}$, for benchmark spin-0 color-octet resonances from Table I, with $\Gamma_{g g}=\Gamma / 5$, and $M_{X}=1000$ (top row), 2000 (middle row), and $3000 \mathrm{GeV}$ (bottom row). The parton-level distributions are shown in the left column panels. These are then smeared by convolution with the estimated detector responses shown in Fig. 7 to obtain the dijet invariant-mass distributions in the right column panels. In all six panels, the red lines show the naive results for the resonant signal $g g \rightarrow X \rightarrow g g$, while the blue lines show the full results including the interferences with the QCD background $g g \rightarrow g g$. 


\section{Monte Carlo results with detector simulation}

Figure 16 shows the dijet invariant-mass distributions for the spin-2 color-singlet resonance benchmarks of Table I with $\Gamma=\Gamma_{g g}$, at the $13 \mathrm{TeV}$ LHC, obtained using Monte Carlo simulations. The results are shown for a digluon resonant process $g g \rightarrow X \rightarrow g g$ and the full results, which also include the interferences with the continuum
QCD $g g \rightarrow g g$ amplitudes for all four color flows shown in Fig. 8.

As was the case with the color-singlet scalars, from Fig. 16 we see that the interference effects have the least impact for the color flow that we call $X_{s}$. On the other hand, interference effects are more pronounced for the $t-/ u$ channel color flow of both QCD and resonant processes.
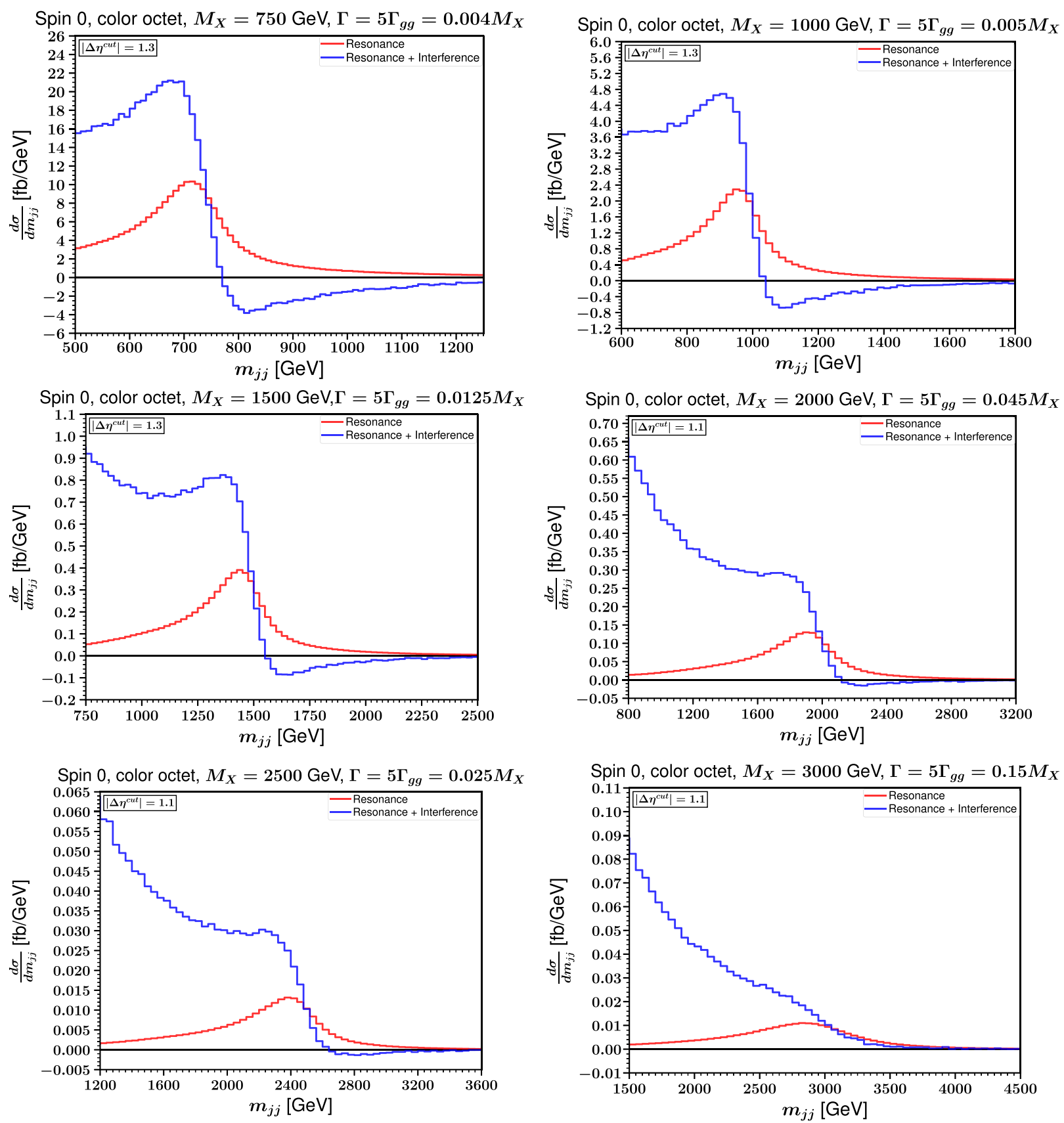

FIG. 20. Dijet invariant-mass distributions for the spin-0 color-octet benchmarks of Table I with $\Gamma_{g g}=\Gamma / 5$, at the $13 \mathrm{TeV}$ LHC, obtained with showering, hadronization, and detector simulation. The red lines show the naive results with only the resonance diagrams of the $g g \rightarrow X \rightarrow g g$ process (RES), which include the $s-, t$, and $u$-channel exchanges of $X$, while the blue lines show the full results including interferences with the continuum QCD $g g \rightarrow g g$ amplitudes (INT). The cases shown in the right column can be compared directly to those in the right column of the previous Fig. 19 based on the more simplistic method of smearing parton level distributions by convolution with an approximate detector response dijet mass distribution. 
Also, we confirm that QCD interferences have a negative cross section for smaller invariant masses $m_{j j} \ll M_{X}$. Then, similar to the case of spin- 0 color-singlet resonances, there is a peak/dip pattern around the resonance mass $m_{j j} \approx M_{X}$, in agreement with the results at parton level with smearing found in Fig. 15. The net effect of negative interference both above and below the $M_{X}$ means that, after fitting to the QCD background, the resonance peak could actually stand out more prominently than predicted by the naive pure-resonance prediction. Once again, the relative importance of the interference for $m_{j j}<M_{X}$ increases with $M_{X}$.

\section{RESULTS FOR $\boldsymbol{\Gamma}=\mathbf{5} \boldsymbol{\Gamma}_{g g}$}

As noted in Ref. [55], the interference effects are expected to be relatively enhanced for smaller branching ratios $\operatorname{BR}(X \rightarrow g g)=\Gamma_{g g} / \Gamma$, for a given fixed resonant
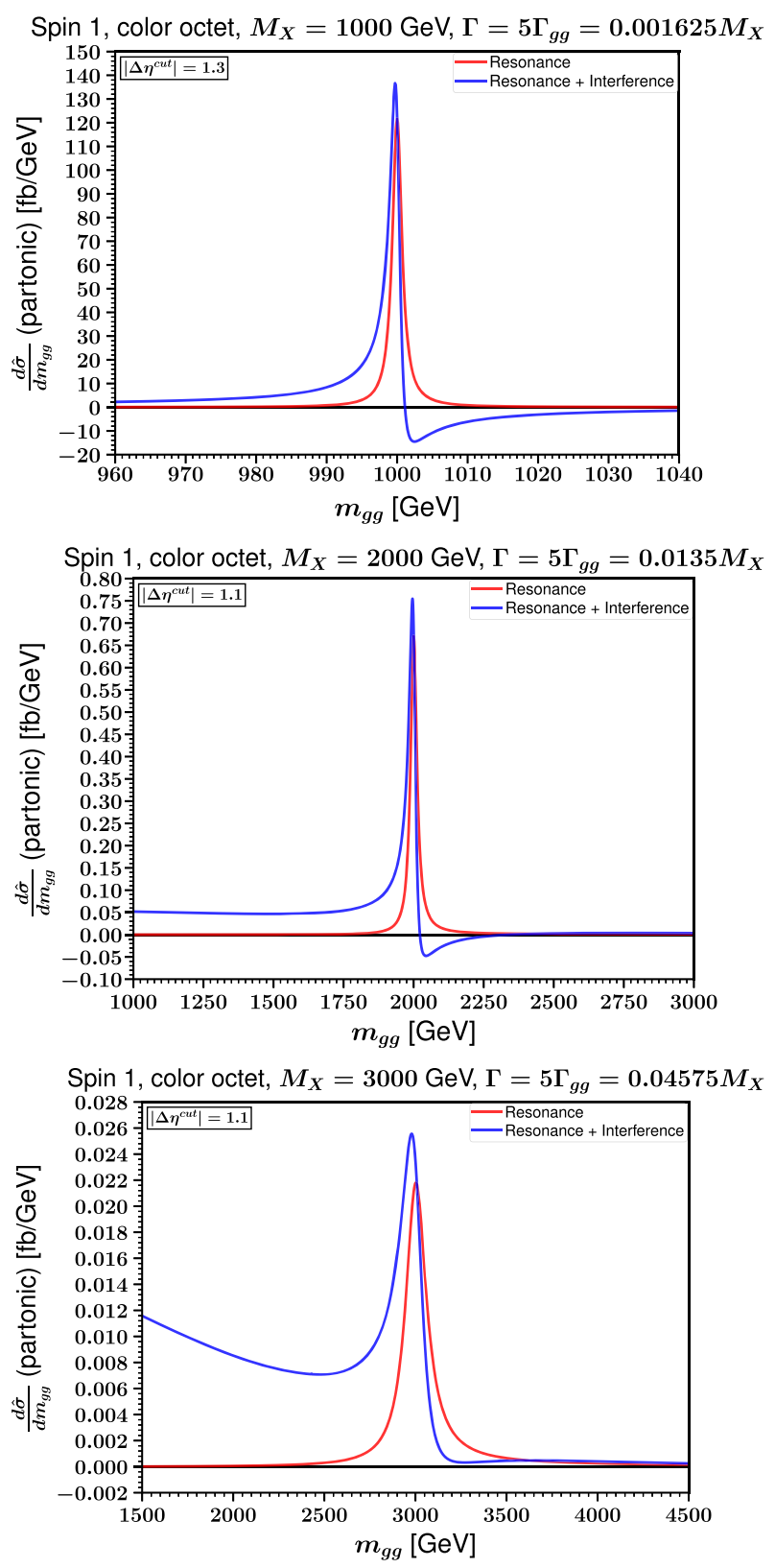
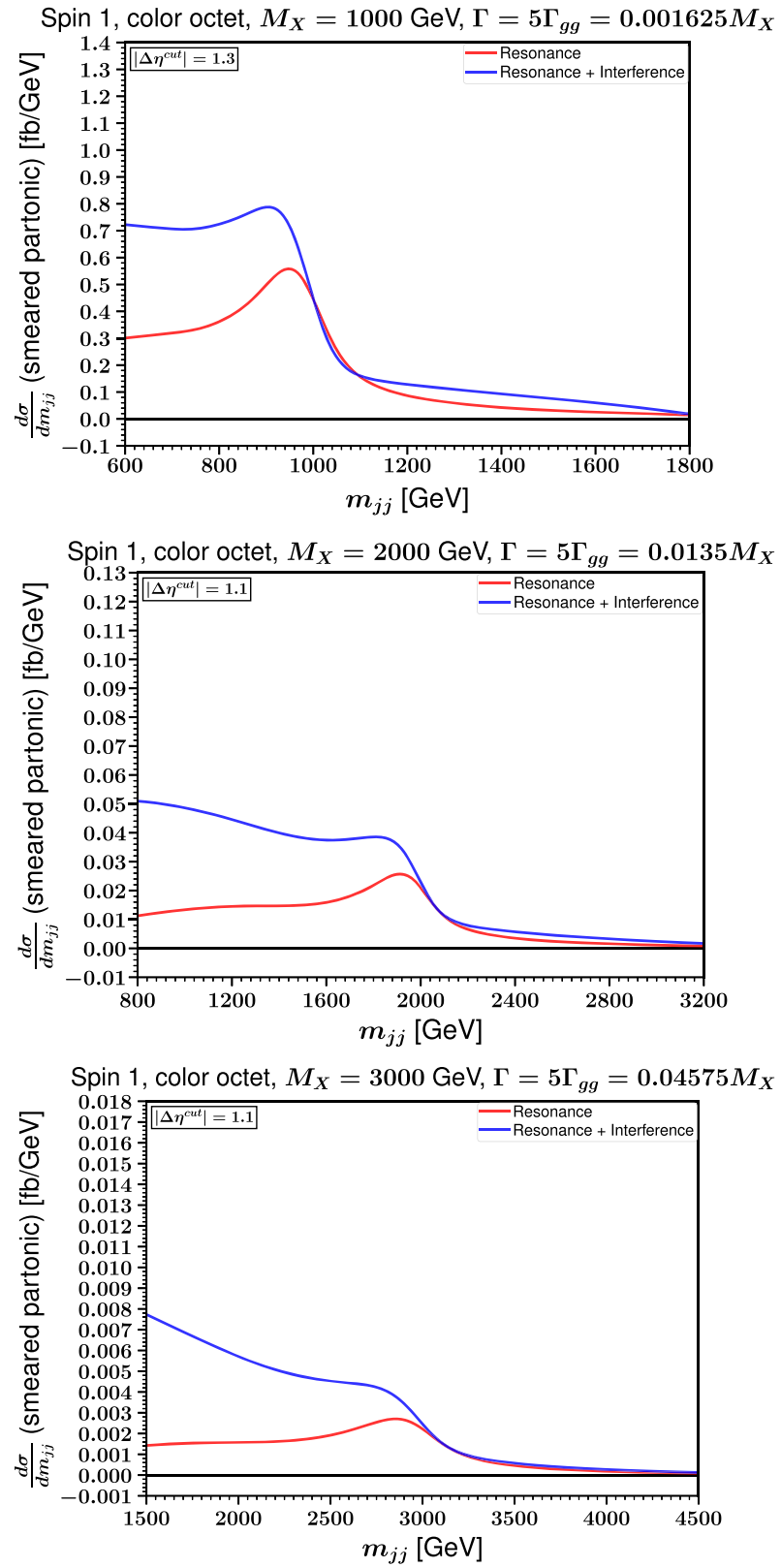

FIG. 21. Digluon invariant-mass distributions, at the $13 \mathrm{TeV}$ LHC, for benchmark spin-1 color-octet resonances from Table I, with $\Gamma_{g g}=\Gamma / 5$, and $M_{X}=1000$ (top row), 2000 (middle row), and $3000 \mathrm{GeV}$ (bottom row). The parton-level distributions are shown in the left column panels. These are then smeared by convolution with the estimated detector responses shown in Fig. 7 to obtain the dijet invariant-mass distributions in the right column panels. In all six panels, the red lines show the naive results for the resonant signal $g g \rightarrow X \rightarrow g g$, while the blue lines show the full results including the interferences with the QCD background $g g \rightarrow g g$. 
production cross section. The reason for this is that to reach the same cross section, both $\Gamma_{g g}$ and $\Gamma$ must be larger than if they were equal [see Eq. (2.21)], leading to much larger Breit-Wigner tails away from the resonance region, which then produce larger interference with the QCD amplitude. Thus, the case with $\Gamma=\Gamma_{g g}$ studied above actually has the smallest impact on the interference with QCD, compared to the general case $\Gamma_{g g}<\Gamma$. In this section, we illustrate this by considering, somewhat arbitrarily, the case that $\Gamma_{g g}=\Gamma / 5$.

The organization of results and structure of the figures below is exactly the same as in the previous section. Thus in Figs. 17, 19, 21, and 23, we show the parton-level distributions before (left columns) and after (right columns) smearing by convolution with the detector response functions illustrated in Fig. 7, for benchmark masses 1000 (top rows),
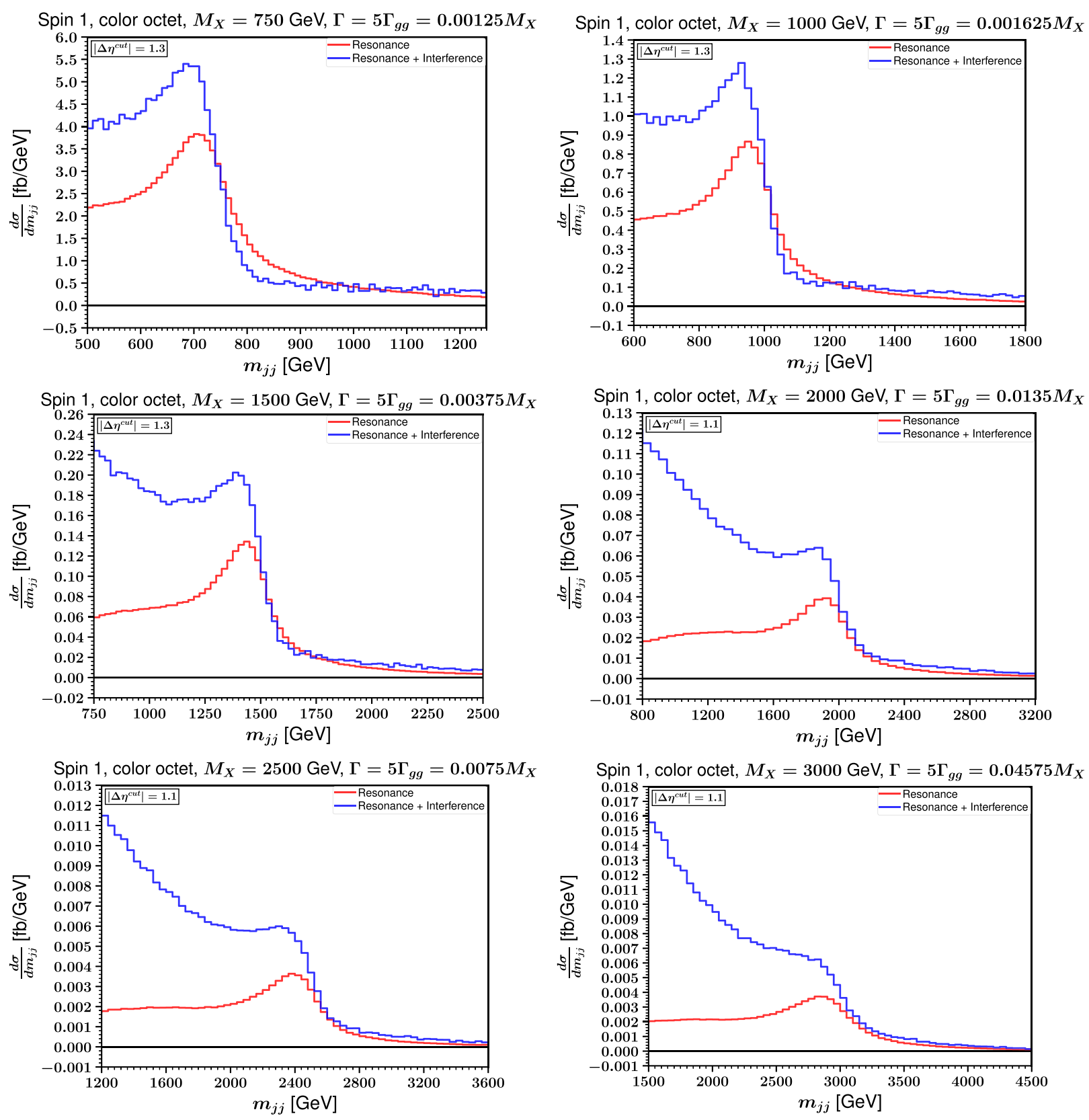

FIG. 22. Dijet invariant-mass distributions for the spin- 1 color-octet benchmarks of Table I with $\Gamma_{g g}=\Gamma / 5$, at the $13 \mathrm{TeV} \mathrm{LHC}$, obtained with showering, hadronization, and detector simulation. The red lines show the naive results with only the resonance diagrams of the $g g \rightarrow X \rightarrow g g$ process (RES), which include the $s-, t$, and $u$-channel exchanges of $X$, while the blue lines show the full results including interferences with the continuum QCD $g g \rightarrow g g$ amplitudes (INT). The cases shown in the right column can be compared directly to those in the right column of the previous Fig. 21 based on the more simplistic method of smearing parton level distributions by convolution with an approximate detector response dijet mass distribution. 
2000 (middle rows), and $3000 \mathrm{GeV}$ (bottom rows). For $\Gamma_{g g}=\Gamma / 5$, the benchmark width-to-mass ratios turn out to be very large for a few cases considered here. For example, $\Gamma / M_{X}=(1.75,0.475)$ for a spin-0 color-singlet with $M_{X}=(3000,2000) \mathrm{GeV}$, and $\Gamma / M_{X}=0.35$ for a spin-2 color-singlet with $M_{X}=3000 \mathrm{GeV}$. The case with $\Gamma / M_{X}=$ 1.75 for a $3000 \mathrm{GeV}$ massive color-singlet (pseudo)scalar has an unrealistically large width-to-mass ratio for a resonance, and is therefore omitted. On the other hand, cases with a large width-to-mass ratio but with $\Gamma / M_{X}<0.55$ are not omitted as the CMS experiment has considered broad resonances with widths up to $55 \%$ of the resonance mass in Ref. [2].

In Figs. 18, 20, 22, and 24, we show the results obtained by our full event simulation including showering, hadronization, and detector simulation. The right columns of pairs of figures (Figs. 17 and 18 for spin-0 color singlet, Figs. 19 and 20 for spin- 0 color octet, Figs. 21 and 22 for
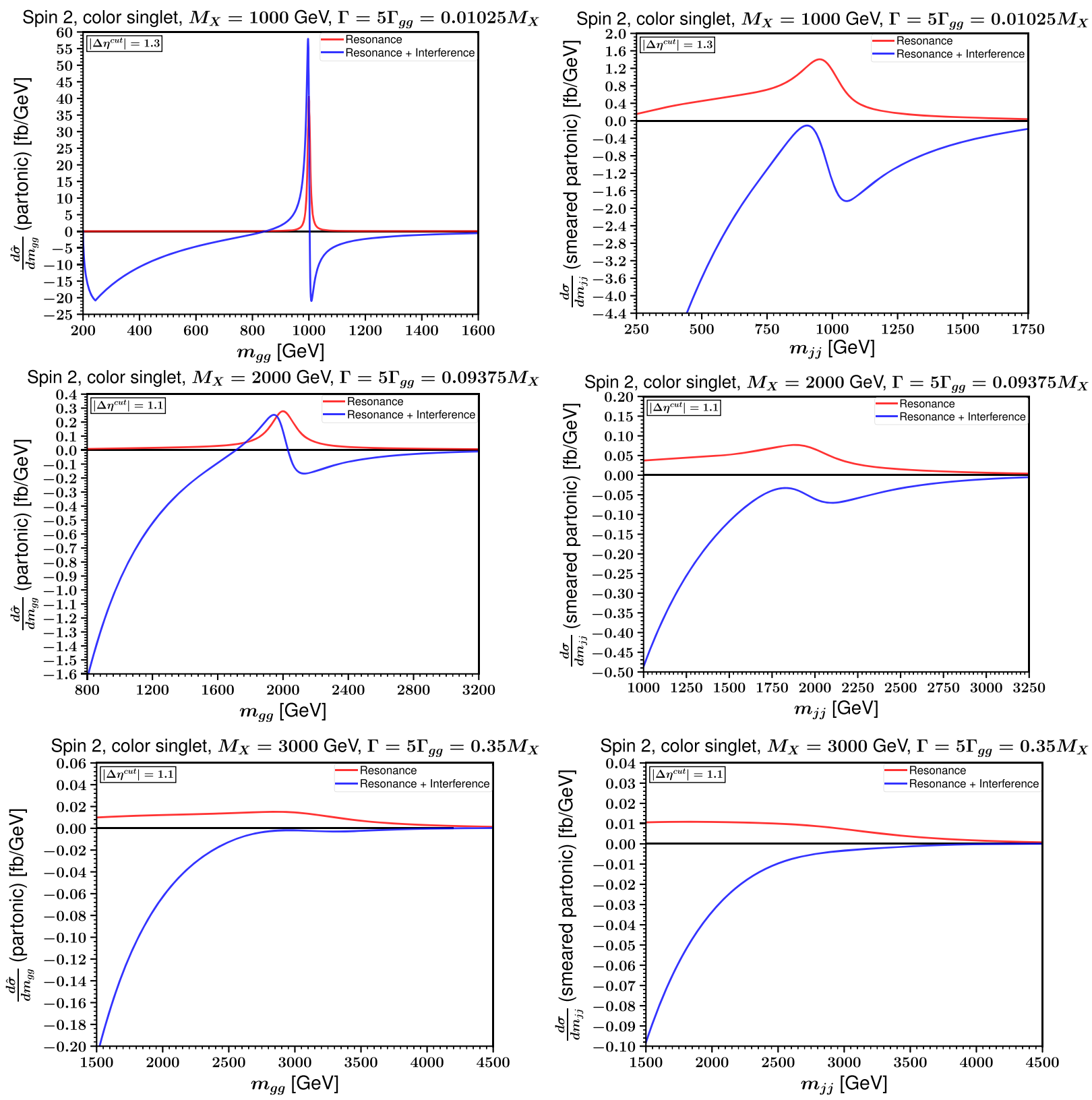

FIG. 23. Digluon invariant-mass distributions, at the $13 \mathrm{TeV}$ LHC, for benchmark spin-2 color-singlet resonances from Table I, with $\Gamma_{g g}=\Gamma / 5$, and $M_{X}=1000$ (top row), 2000 (middle row), and $3000 \mathrm{GeV}$ (bottom row). The parton-level distributions are shown in the left column panels. These are then smeared by convolution with the estimated detector responses shown in Fig. 7 to obtain the dijet invariant-mass distributions in the right column panels. In all six panels, the red lines show the naive results for the resonant signal $g g \rightarrow X \rightarrow g g$, while the blue lines show the full results including the interferences with the QCD background $g g \rightarrow g g$. The negative tails at small invariant mass come from the interference between the QCD amplitudes and the $t$ - and $u$-channel $X$ exchange diagrams. 
spin-1 color octet, and Figs. 23 and 24 for spin-2 color singlet) can be directly compared, as they feature the same masses and widths. The agreement between these sets of figures appears to be good at the qualitative level, but with differing yields at up to the level of tens of percent, and somewhat different shapes in some cases. In particular, for spin 1, the full event simulation produces larger low-mass tails than the smeared parton-level results. In addition, the color-flow choice in the color-singlet interference cases is seen to be a nontrivial effect.

A general feature that can be seen in all of these figures is that when $\Gamma_{g g}=\Gamma / 5$, the distributions including

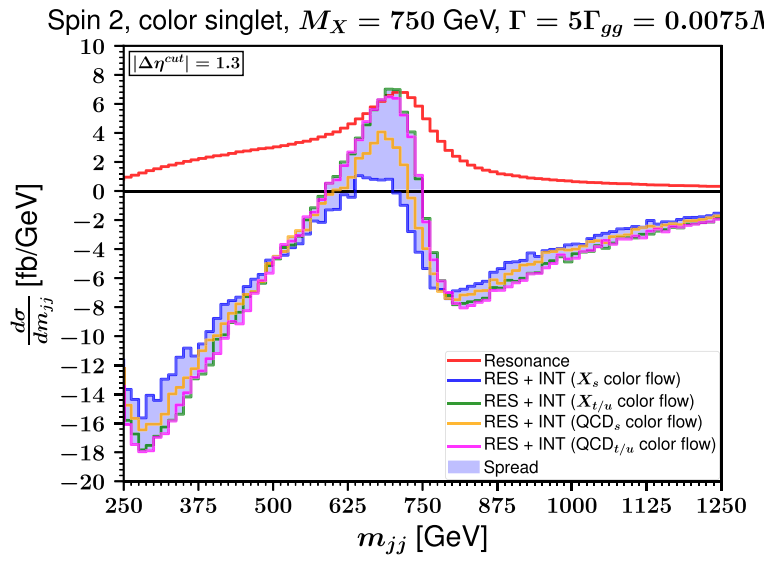

Spin 2, color singlet, $M_{X}=1500 \mathrm{GeV}, \Gamma=5 \Gamma_{g g}=0.025 M_{X}$

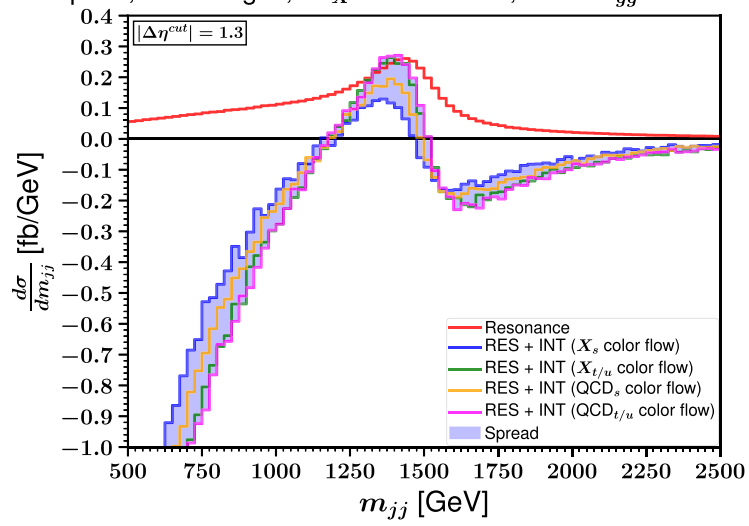

Spin 2, color singlet, $M_{X}=2500 \mathrm{GeV}, \Gamma=5 \Gamma_{g g}=0.055 M_{X}$

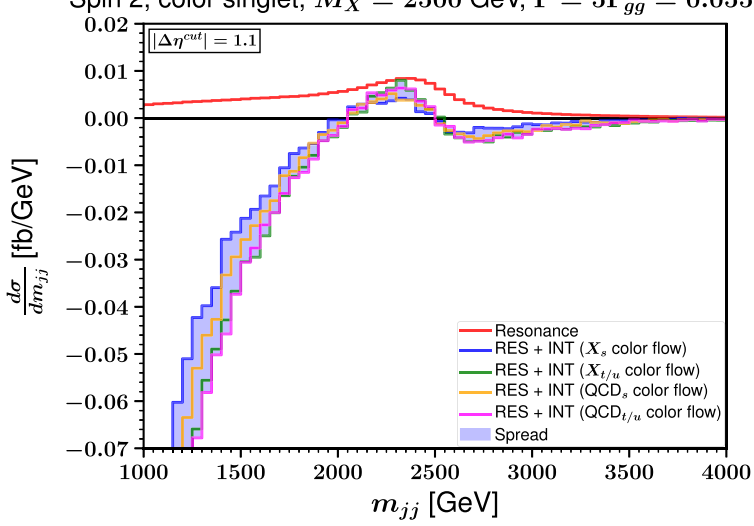

Spin 2, color singlet, $M_{X}=1000 \mathrm{GeV}, \Gamma=5 \Gamma_{g g}=0.01025 M_{X}$

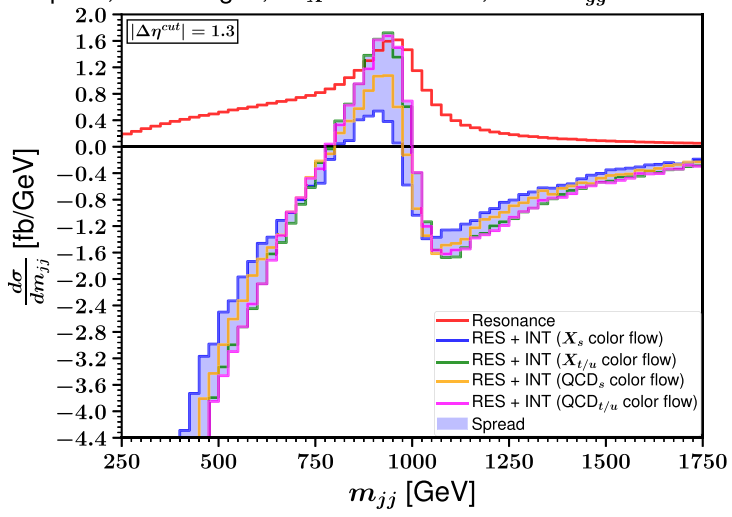

Spin 2, color singlet, $M_{X}=2000 \mathrm{GeV}, \Gamma=5 \Gamma_{g g}=0.09375 M_{X}$
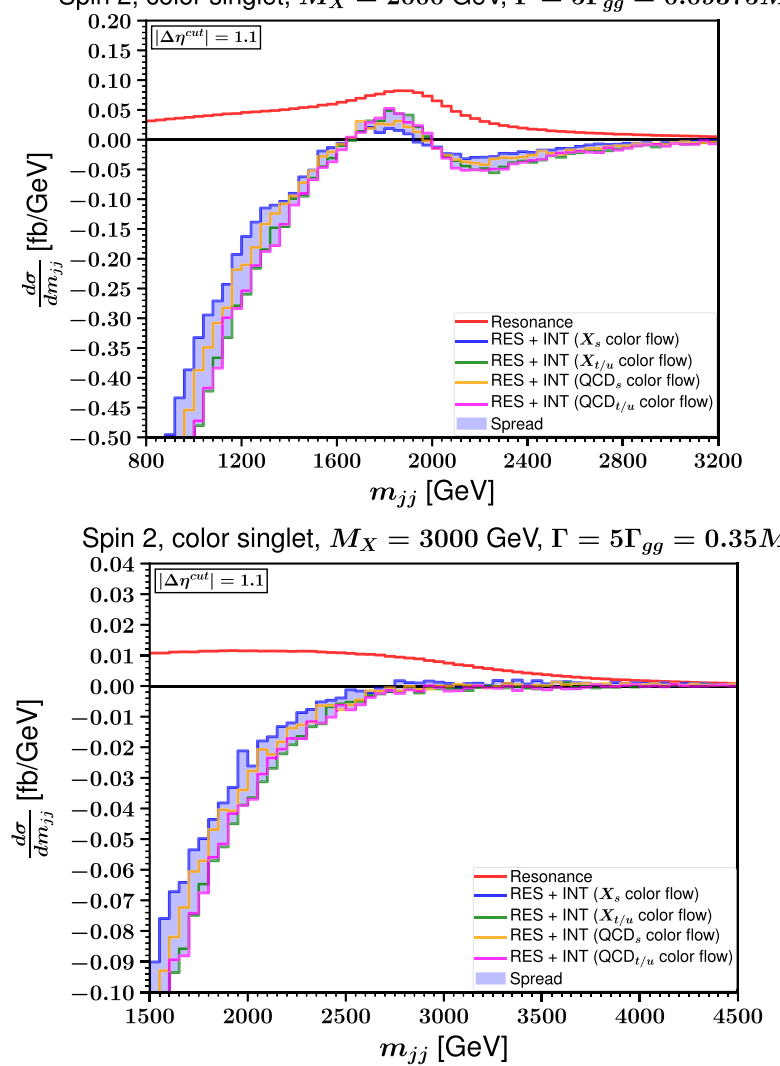

FIG. 24. Dijet invariant-mass distributions for the spin-2 color-singlet benchmarks of Table I with $\Gamma_{g g}=\Gamma / 5$, at the $13 \mathrm{TeV} \mathrm{LHC}$, obtained with showering, hadronization, and detector simulation. The red lines show the naive results with only the resonance diagrams of the $g g \rightarrow X \rightarrow g g$ process (RES), which include the $s-, t$, and $u$-channel exchanges of $X$, while the other four colored lines show the full results including interferences with the continuum QCD $g g \rightarrow g g$ amplitudes (INT) for all four color flows shown in Fig. 8, as labeled. The shaded region shows the spread in the full result in each invariant-mass bin for the different color flow choices. The negative tails at small invariant mass come from the interference between the QCD amplitudes and the $t$ - and $u$-channel $X$ exchange diagrams. The cases shown in the right column can be compared directly to those in the right column of the previous Fig. 23 based on the more simplistic method of smearing parton level distributions by convolution with an approximate detector response dijet mass distribution. 
interference can bear little resemblance to the naive resonance-only results. In each of the spin-0 and spin-1 cases, there is a very large low-mass tail from the interference. For lower $M_{X}$ and spin 0 , we find a pronounced dip for $m_{j j}$ above $M_{X}$, but this tends to be washed out for larger $M_{X}$ and higher spin. In practice, the falling distribution well below $M_{X}$ will be partly absorbed into the QCD background fit and, if present, can therefore have a significant effect on that fit. The result could be a peak/dip or dip shape in the residual fit. We note that the magnitude of the effective dip after background fitting could easily be as large as or larger than the peak that would be naively expected from the resonance if one ignored the interference effect. We also emphasize that the low-mass tail from interference is much larger than the width effect in the pureresonant contribution (visible as the broad distribution of the red curves). The latter effect has been considered in experimental searches [1,2] with $\Gamma>\Gamma_{g g}$, but the much larger former effect has not.

In the spin-2 color-singlet case (see Fig. 24), the distribution shape tends to feature a large deficit at low masses, a peak just below $M_{X}$, and then another deficit above $M_{X}$. After fitting the QCD background, this should lead to an enhancement of the peak compared to the naive resonance-only distribution, so we expect that the actual limits attainable would likely be stronger than those inferred without considering interference.

\section{OUTLOOK}

In this paper, we studied the importance of the interference between the digluon resonant signal and the QCD background amplitude in LHC searches. We showed that the interference terms change the naive Breit-Wigner resonance peak to more like a peak-dip structure around the resonance mass. However, the particular characteristic shape depends on the spin and the color of the digluon resonance. The interference effects were studied for scalar and pseudoscalar resonances in both singlet and octet color representations, spin-1 color octets, and color-singlet massive gravitons. To show the importance of the interference effects, we considered a few benchmark examples for various resonance masses, such that their production cross sections are close to the claimed exclusions of the CMS experiment in Refs. [1,2].

We found that the effects of interference were larger for the spin- 0 color-singlet case than for the spin- 0 color-octet and spin-1 color-octet cases, as was expected from consideration of the parton-level differential cross sections. We also note that the relative impact of the interference stays nearly constant for a fixed $M_{X}$ as the resonant cross section decreases, but tends to increase for larger $M_{X}$. It can also increase dramatically if the resonance has other decays contributing to its width that are not detectable for some reason. Our results still contain significant uncertainties, in particular from the color-flow ambiguities in the color-singlet cases, and from the fact that in this paper we have not included any NLO effects. It would be interesting to go beyond the approximations used in this paper in order to reduce these sources of uncertainty.

After performing a fit to the QCD background, the residual signal for a digluon resonance could have a shape in the invariant-mass distribution that appears to resemble a peak/dip, a shelf, an enhanced peak, or even a pure dip. For all cases except spin 2, there is a large positive tail at invariant masses below $M_{X}$. Because the magnitude of the QCD background amplitude falls with $\sqrt{\hat{s}}$, the low-mass tail tends to be more significant than the high-mass deficit from the interference. In the spin-2 case, we found that the low-mass tail is larger in magnitude but actually switches sign, and is negative far below $M_{X}$, due to interference between the QCD background and the $t$ - and $u$-channel $X$ exchange. After QCD fitting and subtraction, this could lead to an enhanced peak in the spin-2 case, compared to the naive pure-resonance distribution expected if one neglects the interference.

More generally, the results found here point to the appropriateness of a flexible approach to searching for dijet resonances. Although one is searching for a resonance, we have seen that there is a considerable diversity of possible invariant-mass distribution signals even for resonances with rather narrow widths, depending on the resonance quantum numbers, width, and branching ratios, as can be seen from the figures above. Perhaps advanced data analysis and machine learning techniques can be brought to bear on the problem of identifying or setting limits on new-physics anomalies in mass distributions in a general and efficient way (see, for example, Refs. [120-124] for recent developments). In any case, it seems necessary to consider a variety of possible anomalies in the dijet mass distribution, without undue prejudice towards a simple resonance peak.

\section{ACKNOWLEDGMENTS}

P. N. B. thanks Olivier Mattelaer, Richard Efrain Ruiz, and Leif Gellersen for helpful discussions at MADGRAPH School (India). P. N. B. thanks Sergey A. Uzunyan for his help and support in using the NICADD compute cluster at Northern Illinois University. P. N. B. also thanks Jeremiah Mitchell, Manoj Kumar Mandal, and Chien-Yi Chen for technical advice. S. P. M. thanks Zhen Liu for helpful discussions. This work was supported in part by the National Science Foundation Grant No. PHY-1719273. 
[1] A. M. Sirunyan et al. (CMS Collaboration), Search for narrow and broad dijet resonances in proton-proton collisions at $\sqrt{s}=13 \mathrm{TeV}$ and constraints on dark matter mediators and other new particles, J. High Energy Phys. 08 (2018) 130.

[2] A. M. Sirunyan et al. (CMS Collaboration), Search for high mass dijet resonances with a new background prediction method in proton-proton collisions at $\sqrt{s}=13 \mathrm{TeV}$, J. High Energy Phys. 05 (2020) 033.

[3] M. Aaboud et al. (ATLAS Collaboration), Search for new phenomena in dijet events using $37 \mathrm{fb}^{-1}$ of $p p$ collision data collected at $\sqrt{s}=13 \mathrm{TeV}$ with the ATLAS detector, Phys. Rev. D 96, 052004 (2017).

[4] G. Aad et al. (ATLAS Collaboration), Search for new resonances in mass distributions of jet pairs using $139 \mathrm{fb}^{-1}$ of $p p$ collisions at $\sqrt{s}=13 \mathrm{TeV}$ with the ATLAS detector, J. High Energy Phys. 03 (2020) 145.

[5] S. Chatrchyan et al. (CMS Collaboration), Search for narrow resonances using the dijet mass spectrum in $p p$ collisions at $\sqrt{s}=8 \mathrm{TeV}$, Phys. Rev. D 87, 114015 (2013).

[6] G. Aad et al. (ATLAS Collaboration), Search for new phenomena in the dijet mass distribution using $p-p$ collision data at $\sqrt{s}=8 \mathrm{TeV}$ with the ATLAS detector, Phys. Rev. D 91, 052007 (2015).

[7] V. Khachatryan et al. (CMS Collaboration), Search for resonances and quantum black holes using dijet mass spectra in proton-proton collisions at $\sqrt{s}=8 \mathrm{TeV}$, Phys. Rev. D 91, 052009 (2015).

[8] V. Khachatryan et al. (CMS Collaboration), Search for Narrow Resonances in Dijet Final States at $\sqrt{(} s)=8 \mathrm{TeV}$ with the Novel CMS Technique of Data Scouting, Phys. Rev. Lett. 117, 031802 (2016).

[9] G. Aad et al. (ATLAS Collaboration), Search for new phenomena in dijet mass and angular distributions from $p p$ collisions at $\sqrt{s}=13 \mathrm{TeV}$ with the ATLAS detector, Phys. Lett. B 754, 302 (2016).

[10] V. Khachatryan et al. (CMS Collaboration), Search for Narrow Resonances Decaying to Dijets in Proton-Proton Collisions at $\sqrt{(} s)=13 \mathrm{TeV}$, Phys. Rev. Lett. 116, 071801 (2016).

[11] A. M. Sirunyan et al. (CMS Collaboration), Search for dijet resonances in proton-proton collisions at $\sqrt{s}=13 \mathrm{TeV}$ and constraints on dark matter and other models, Phys. Lett. B 769, 520 (2017); Erratum, Phys. Lett. B 772, 882 (2017).

[12] P. H. Frampton and S. L. Glashow, Chiral color: An alternative to the standard model, Phys. Lett. B 190, 157 (1987).

[13] P. H. Frampton and S. L. Glashow, Unifiable Chiral Color with Natural GIM Mechanism, Phys. Rev. Lett. 58, 2168 (1987).

[14] P. H. Frampton, Alternative version of chiral color as alternative to the standard model, Phys. Rev. D 81, 095005 (2010).

[15] J. C. Pati and A. Salam, Mirror fermions, $J / \psi$ particles, Kolar mine events and neutrino anomaly, Phys. Lett. 58B, 333 (1975).

[16] J. Preskill, Subgroup alignment in hypercolor theories, Nucl. Phys. B177, 21 (1981).
[17] L. J. Hall and A. E. Nelson, Heavy gluons and monojets, Phys. Lett. 153B, 430 (1985).

[18] J. Bagger, C. Schmidt, and S. King, Axigluon production in hadronic collisions, Phys. Rev. D 37, 1188 (1988).

[19] R. S. Chivukula, A. G. Cohen, and E. H. Simmons, New strong interactions at the tevatron? Phys. Lett. B 380, 92 (1996).

[20] E. H. Simmons, Coloron phenomenology, Phys. Rev. D 55, 1678 (1997).

[21] Y. Bai and B. A. Dobrescu, Heavy octets and tevatron signals with three or four b jets, J. High Energy Phys. 07 (2011) 100.

[22] R. S. Chivukula, A. Farzinnia, J. Ren, and E. H. Simmons, Constraints on the scalar sector of the renormalizable coloron model, Phys. Rev. D 88, 075020 (2013); Erratum, Phys. Rev. D 89, 059905 (2014).

[23] R. S. Chivukula, A. Farzinnia, J. Ren, and E. H. Simmons, Hadron collider production of massive color-octet vector bosons at next-to-leading order, Phys. Rev. D 87, 094011 (2013).

[24] R. S. Chivukula, E. H. Simmons, A. Farzinnia, and J. Ren, LHC constraints on a Higgs boson partner from an extended color sector, Phys. Rev. D 90, 015013 (2014).

[25] C. Y. Chen, A. Freitas, T. Han, and K. S. M. Lee, Heavy color-octet particles at the LHC, J. High Energy Phys. 05 (2015) 135.

[26] R. S. Chivukula, A. Farzinnia, and E. H. Simmons, Vacuum stability and triviality analyses of the renormalizable coloron model, Phys. Rev. D 92, 055002 (2015).

[27] Y. Bai and B. A. Dobrescu, Minimal $S U(3) \times S U(3)$ symmetry breaking patterns, Phys. Rev. D 97, 055024 (2018).

[28] P. Agrawal and K. Howe, Factoring the strong $C P$ problem, J. High Energy Phys. 12 (2018) 029.

[29] Y. Bai and B. A. Dobrescu, Collider tests of the renormalizable coloron model, J. High Energy Phys. 04 (2018) 114.

[30] Y. Bai, S. Lu, and Q. F. Xiang, Hexapod coloron at the LHC, J. High Energy Phys. 08 (2018) 200.

[31] S. P. Martin, Mixed gluinos and sgluons from a new $S U(3)$ gauge group, Phys. Rev. D 101, 035019 (2020).

[32] C. T. Hill, Topcolor: Top quark condensation in a gauge extension of the standard model, Phys. Lett. B 266, 419 (1991).

[33] S. P. Martin, Renormalizable top quark condensate models, Phys. Rev. D 45, 4283 (1992); A tumbling top quark condensate model, Phys. Rev. D 46, 2197 (1992).

[34] C. T. Hill and S. J. Parke, Top production: Sensitivity to new physics, Phys. Rev. D 49, 4454 (1994).

[35] B. A. Dobrescu and C. T. Hill, Electroweak Symmetry Breaking Via Top Condensation Seesaw, Phys. Rev. Lett. 81, 2634 (1998).

[36] R. S. Chivukula, B. A. Dobrescu, H. Georgi, and C. T. Hill, Top quark Seesaw theory of electroweak symmetry breaking, Phys. Rev. D 59, 075003 (1999).

[37] J. L. Hewett and T. G. Rizzo, Low-energy phenomenology of superstring inspired E(6) models, Phys. Rep. 183, 193 (1989).

[38] E. Eichten, I. Hinchliffe, K. D. Lane, and C. Quigg, Super collider physics, Rev. Mod. Phys. 56, 579 (1984); Rev. Mod. Phys. 58, 1065(A) (1986). 
[39] B. A. Dobrescu and F. Yu, Coupling-mass mapping of dijet peak searches, Phys. Rev. D 88, 035021 (2013); Erratum, Phys. Rev. D 90, 079901 (2014).

[40] U. Baur, I. Hinchliffe, and D. Zeppenfeld, Excited quark production at hadron colliders, Int. J. Mod. Phys. A 02, 1285 (1987).

[41] U. Baur, M. Spira, and P. M. Zerwas, Excited quark and lepton production at hadron colliders, Phys. Rev. D 42, 815 (1990).

[42] L. A. Anchordoqui, H. Goldberg, D. Lust, S. Nawata, S. Stieberger, and T. R. Taylor, Dijet Signals for Low Mass Strings at the LHC, Phys. Rev. Lett. 101, 241803 (2008).

[43] S. Cullen, M. Perelstein, and M. E. Peskin, TeV strings and collider probes of large extra dimensions, Phys. Rev. D 62, 055012 (2000).

[44] C. T. Hill and E. H. Simmons, Strong dynamics and electroweak symmetry breaking, Phys. Rep. 381, 235 (2003); Erratum, Phys. Rep. 390, 553 (2004).

[45] B. A. Dobrescu, K. Kong, and R. Mahbubani, Leptons and photons at the LHC: Cascades through spinless adjoints, J. High Energy Phys. 07 (2007) 006.

[46] B. A. Dobrescu, K. Kong, and R. Mahbubani, Massive color-octet bosons and pairs of resonances at hadron colliders, Phys. Lett. B 670, 119 (2008).

[47] L. Randall and R. Sundrum, An Alternative to Compactification, Phys. Rev. Lett. 83, 4690 (1999).

[48] T. Han, J. D. Lykken, and R. J. Zhang, On Kaluza-Klein states from large extra dimensions, Phys. Rev. D 59, 105006 (1999).

[49] M. Chala, F. Kahlhoefer, M. McCullough, G. Nardini, and K. Schmidt-Hoberg, Constraining dark sectors with monojets and dijets, J. High Energy Phys. 07 (2015) 089.

[50] D. Abercrombie et al., Dark matter benchmark models for early LHC Run-2 searches: Report of the ATLAS/CMS dark matter forum, Phys. Dark Univ. 26, 100371 (2019).

[51] J. Abdallah et al., Simplified models for dark matter searches at the LHC, Phys. Dark Universe 9-10, 8 (2015).

[52] A. Boveia et al., Recommendations on presenting LHC searches for missing transverse energy signals using simplified $s$-channel models of dark matter, Phys. Dark Univ. 27, 100365 (2020).

[53] T. Han, I. Lewis, and Z. Liu, Colored resonant signals at the LHC: Largest rate and simplest topology, J. High Energy Phys. 12 (2010) 085.

[54] S. Mukherjee (CMS Collaboration), Data scouting: A new trigger paradigm, arXiv:1708.06925.

[55] S. P. Martin, Signal-background interference for a singlet spin-0 digluon resonance at the LHC, Phys. Rev. D 94, 035003 (2016).

[56] D. A. Dicus and S. S. D. Willenbrock, Photon pair production and the intermediate mass Higgs boson, Phys. Rev. D 37, 1801 (1988).

[57] L. J. Dixon and M. S. Siu, Resonance Continuum Interference in the Diphoton Higgs Signal at the LHC, Phys. Rev. Lett. 90, 252001 (2003).

[58] S. P. Martin, Shift in the LHC Higgs diphoton mass peak from interference with background, Phys. Rev. D 86, 073016 (2012).

[59] D. de Florian, N. Fidanza, R. J. Hernandez-Pinto, J. Mazzitelli, Y. Rotstein Habarnau, and G. F. R. Sborlini,
A complete $O\left(\alpha_{S}^{2}\right)$ calculation of the signal-background interference for the Higgs diphoton decay channel, Eur. Phys. J. C 73, 2387 (2013).

[60] S. P. Martin, Interference of Higgs diphoton signal and background in production with a jet at the LHC, Phys. Rev. D 88, 013004 (2013).

[61] L. J. Dixon and Y. Li, Bounding the Higgs Boson Width Through Interferometry, Phys. Rev. Lett. 111, 111802 (2013).

[62] F. Coradeschi, D. de Florian, L. J. Dixon, N. Fidanza, S. Hche, H. Ita, Y. Li, and J. Mazzitelli, Interference effects in the $H(\rightarrow \gamma \gamma)+2$ jets channel at the LHC, Phys. Rev. D 92, 013004 (2015).

[63] C. P. Becot, Diphoton lineshape of the BEH boson using the ATLAS detector at the LHC: Calibration, mass, width and interferences, CERN-THESIS-2015-193, LAL-15-303.

[64] J. Campbell, M. Carena, R. Harnik, and Z. Liu, Interference in the $g g \rightarrow h \rightarrow \gamma \gamma$ On-Shell Rate and the Higgs Boson Total Width, Phys. Rev. Lett. 119, 181801 (2017).

[65] L. Cieri, F. Coradeschi, D. de Florian, and N. Fidanza, Transverse-momentum resummation for the signalbackground interference in the $H \rightarrow \gamma \gamma$ channel at the LHC, Phys. Rev. D 96, 054003 (2017).

[66] E. W. N. Glover and J. J. van der Bij, Vector boson pair production via gluon fusion, Phys. Lett. B 219, 488 (1989).

[67] E. W. N. Glover and J. J. van der Bij, Z boson pair production via gluon fusion, Nucl. Phys. B321, 561 (1989).

[68] M. H. Seymour, The Higgs boson line shape and perturbative unitarity, Phys. Lett. B 354, 409 (1995).

[69] T. Binoth, M. Ciccolini, N. Kauer, and M. Kramer, Gluoninduced W-boson pair production at the LHC, J. High Energy Phys. 12 (2006) 046.

[70] E. Accomando, The process $g g \rightarrow W W$ as a probe into the EWSB mechanism, Phys. Lett. B 661, 129 (2008).

[71] J. M. Campbell, R. K. Ellis, and C. Williams, Gluon-gluon contributions to $W^{+} W^{-}$production and Higgs interference effects, J. High Energy Phys. 10 (2011) 005.

[72] N. Kauer, Signal-background interference in $g g \rightarrow H \rightarrow V V$, Proc. Sci., RADCOR2011 (2011) 027.

[73] G. Passarino, Higgs interference effects in $g g \rightarrow Z Z$ and their uncertainty, J. High Energy Phys. 08 (2012) 146.

[74] N. Kauer and G. Passarino, Inadequacy of zero-width approximation for a light Higgs boson signal, J. High Energy Phys. 08 (2012) 116; N. Kauer, Inadequacy of zero-width approximation for a light Higgs boson signal, Mod. Phys. Lett. A 28, 1330015 (2013).

[75] F. Campanario, Q. Li, M. Rauch, and M. Spira, ZZ + jet production via gluon fusion at the LHC, J. High Energy Phys. 06 (2013) 069.

[76] M. Bonvini, F. Caola, S. Forte, K. Melnikov, and G. Ridolfi, Signal-background interference effects for $g g \rightarrow$ $H \rightarrow W^{+} W^{-}$beyond leading order, Phys. Rev. D 88, 034032 (2013).

[77] F. Caola and K. Melnikov, Constraining the Higgs boson width with ZZ production at the LHC, Phys. Rev. D 88, 054024 (2013).

[78] J. M. Campbell, R. K. Ellis, and C. Williams, Bounding the Higgs width at the LHC using full analytic results for $g g \rightarrow e^{-} e^{+} \mu^{-} \mu^{+}$, J. High Energy Phys. 04 (2014) 060. 
[79] M. Chen, T. Cheng, J. S. Gainer, A. Korytov, K. T. Matchev, P. Milenovic, G. Mitselmakher, M. Park, A. Rinkevicius, and M. Snowball, The role of interference in unraveling the $Z Z$-couplings of the newly discovered boson at the LHC, Phys. Rev. D 89, 034002 (2014).

[80] N. Kauer, Interference effects for $H \rightarrow W W / \mathrm{ZZ} \rightarrow \ell \bar{\nu}_{\ell} \bar{\ell} \nu_{\ell}$ searches in gluon fusion at the LHC, J. High Energy Phys. 12 (2013) 082.

[81] J. M. Campbell, R. K. Ellis, and C. Williams, Bounding the Higgs width at the LHC: Complementary results from $H \rightarrow W W$, Phys. Rev. D 89, 053011 (2014).

[82] C. Englert and M. Spannowsky, Limitations and opportunities of off-shell coupling measurements, Phys. Rev. D 90, 053003 (2014).

[83] J. M. Campbell, R. K. Ellis, E. Furlan, and R. Röntsch, Interference effects for Higgs boson mediated $Z$-pair plus jet production, Phys. Rev. D 90, 093008 (2014).

[84] C. Englert, Y. Soreq, and M. Spannowsky, Off-Shell Higgs Coupling Measurements in BSM scenarios, J. High Energy Phys. 05 (2015) 145.

[85] H. E. Logan, Hiding a Higgs width enhancement from offshell $g g\left(\rightarrow h^{*}\right) \rightarrow$ ZZ measurements, Phys. Rev. D 92, 075038 (2015).

[86] N. Kauer, Off-shell Higgs signal and total width determination at the LHC, Proc. Sci., FFP14 (2016) 114.

[87] C. S. Li, H. T. Li, D. Y. Shao, and J. Wang, Soft gluon resummation in the signal-background interference process of $g g\left(\rightarrow h^{*}\right) \rightarrow Z Z$, J. High Energy Phys. 08 (2015) 065.

[88] V. Khachatryan et al. (CMS Collaboration), Constraints on the Higgs boson width from off-shell production and decay to Z-boson pairs, Phys. Lett. B 736, 64 (2014).

[89] G. Aad et al. (ATLAS Collaboration), Constraints on the off-shell Higgs boson signal strength in the high-mass $Z Z$ and $W W$ final states with the ATLAS detector, Eur. Phys. J. C 75, 335 (2015).

[90] V. Khachatryan et al. (CMS Collaboration), Limits on the Higgs boson lifetime and width from its decay to four charged leptons, Phys. Rev. D 92, 072010 (2015).

[91] V. Khachatryan et al. (CMS Collaboration), Search for Higgs boson off-shell production in proton-proton collisions at 7 and $8 \mathrm{TeV}$ and derivation of constraints on its total decay width, J. High Energy Phys. 09 (2016) 051.

[92] M. Aaboud et al. (ATLAS Collaboration), Constraints on off-shell Higgs boson production and the Higgs boson total width in $Z Z \rightarrow 4 \ell$ and $Z Z \rightarrow 2 \ell 2 \nu$ final states with the ATLAS detector, Phys. Lett. B 786, 223 (2018).

[93] A. M. Sirunyan et al. (CMS Collaboration), Measurements of the Higgs boson width and anomalous $H V V$ couplings from on-shell and off-shell production in the four-lepton final state, Phys. Rev. D 99, 112003 (2019).

[94] E. Boos, V. Bunichev, L. Dudko, and M. Perfilov, Interference between $W^{\prime}$ and $W$ in single-top quark production processes, Phys. Lett. B 655, 245 (2007); T. G. Rizzo, The determination of the helicity of $W^{\prime}$ boson couplings at the LHC, J. High Energy Phys. 05 (2007) 037; A. Papaefstathiou and O. Latunde-Dada, NLO production of $W^{\prime}$ bosons at hadron colliders using the MC@NLO and POWHEG methods, J. High Energy Phys. 07 (2009) 044; E. Accomando, D. Becciolini, S. De Curtis, D. Dominici,
L. Fedeli, and C. Shepherd-Themistocleous, Interference effects in heavy W'-boson searches at the LHC, Phys. Rev. D 85, 115017 (2012); L. Bian, D. Liu, J. Shu, and Y. Zhang, Interference effect on resonance studies in searches of heavy Particles, Int. J. Mod. Phys. 31, 1650083 (2016).

[95] E. Accomando, D. Becciolini, A. Belyaev, S. Moretti, and C. Shepherd-Themistocleous, $Z^{\prime}$ at the LHC: Interference and finite width effects in Drell-Yan, J. High Energy Phys. 10 (2013) 153; E. Accomando, D. Barducci, S. De Curtis, J. Fiaschi, S. Moretti, and C. H. Shepherd-Themistocleous, Drell-Yan production of multi Z'-bosons at the LHC within non-universal ED and 4D composite Higgs models, J. High Energy Phys. 07 (2016) 068.

[96] D. Dicus, A. Stange, and S. Willenbrock, Higgs decay to top quarks at hadron colliders, Phys. Lett. B 333, 126 (1994); D. Choudhury, R. M. Godbole, R. K. Singh, and K. Wagh, Top production at the Tevatron/LHC and nonstandard, strongly interacting spin one particles, Phys. Lett. B 657, 69 (2007); A. Djouadi, G. Moreau, and R. K. Singh, Kaluza-Klein excitations of gauge bosons at the LHC, Nucl. Phys. B797, 1 (2008); R. Frederix and F. Maltoni, Top pair invariant mass distribution: A window on new physics, J. High Energy Phys. 01 (2009) 047; U. Haisch and S. Westhoff, Massive color-octet bosons: Bounds on effects in top-quark pair production, J. High Energy Phys. 08 (2011) 088; N. Craig, F. D'Eramo, P. Draper, S. Thomas, and H. Zhang, The hunt for the rest of the Higgs bosons, J. High Energy Phys. 06 (2015) 137; B. Hespel, F. Maltoni, and E. Vryonidou, Signal background interference effects in heavy scalar production and decay to a topanti-top pair, J. High Energy Phys. 10 (2016) 016; M. Carena and Z. Liu, Challenges and opportunities for heavy scalar searches in the $t \bar{t}$ channel at the LHC, J. High Energy Phys. 11 (2016) 159; A. Djouadi, J. Ellis, and J. Quevillon, Interference effects in the decays of spin-zero resonances into $\gamma \gamma$ and $t \bar{t}$, J. High Energy Phys. 07 (2016) 105; A. Djouadi, J. Ellis, A. Popov, and J. Quevillon, Interference effects in $t \bar{t}$ production at the LHC as a window on new physics, J. High Energy Phys. 03 (2019) 119.

[97] S. Jung, Y. W. Yoon, and J. Song, Interference effect on a heavy Higgs resonance signal in the $\gamma \gamma$ and $Z Z$ channels, Phys. Rev. D 93, 055035 (2016).

[98] S. Jung, J. Song, and Y. W. Yoon, Dip or nothingness of a Higgs resonance from the interference with a complex phase, Phys. Rev. D 92, 055009 (2015).

[99] N. Craig, S. Renner, and D. Sutherland, Exploring peaks and valleys in the diphoton spectrum, Phys. Rev. D 94, 075011 (2016).

[100] N. Kauer, A. Lind, P. Maierhofer, and W. Song, Higgs interference effects at the one-loop level in the 1-HiggsSinglet extension of the Standard Model, J. High Energy Phys. 07 (2019) 108.

[101] D. Choudhury, R. M. Godbole, and P. Saha, Dijet resonances, widths and all that, J. High Energy Phys. 01 (2012) 155.

[102] J. P. Ma, J. X. Wang, and S. Zhao, Breakdown of QCD factorization for P-wave quarkonium production at Low transverse momentum, Phys. Lett. B 737, 103 (2014).

[103] W. Beenakker, R. Kleiss, and G. Lustermans, No LandauYang in QCD, arXiv:1508.07115. 
[104] M. Cacciari, L. Del Debbio, J. R. Espinosa, A. D. Polosa, and M. Testa, A note on the fate of the Landau-Yang theorem in non-Abelian gauge theories, Phys. Lett. B 753, 476 (2016).

[105] Y. Bai and W. Y. Keung, Can vanishing mass-on-shell interactions generate a dip at colliders? Int. J. Mod. Phys. A 30, 1550120 (2015).

[106] M. D. Schwartz, Quantum Field Theory and the Standard Model (Cambridge University Press, New York, 2014).

[107] K. Hagiwara, J. Kanzaki, Q. Li, and K. Mawatari, HELAS and Madgraph/Madevent with spin-2 particles, Eur. Phys. J. C 56, 435 (2008).

[108] G. F. Giudice, R. Rattazzi, and J.D. Wells, Quantum gravity and extra dimensions at high-energy colliders, Nucl. Phys. B544, 3 (1999).

[109] H.E. Haber, Useful relations among the generators in the defining and adjoint representations of $S U(N)$, arXiv: 1912.13302.

[110] A. J. MacFarlane, A. Sudbery, and P. H. Weisz, On Gell-Mann's lambda-matrices, d- and f-tensors, octets, and parametrizations of SU(3), Commun. Math. Phys. 11, 77 (1968).

[111] R. D. Ball, V. Bertone, S. Carrazza, L. Del Debbio, S. Forte, A. Guffanti, N. P. Hartland, and J. Rojo (NNPDF Collaboration), Parton distributions with QED corrections, Nucl. Phys. B877, 290 (2013).

[112] T. Sjostrand, S. Mrenna, and P.Z. Skands, PYTHIA6.4 Physics and Manual, J. High Energy Phys. 05 (2006) 026.

[113] T. Sjostrand, S. Ask, J. R. Christiansen, R. Corke, N. Desai, P. Ilten, S. Mrenna, S. Prestel, C. O. Rasmussen, and P. Z. Skands, An Introduction to PYTHIA8.2, Comput. Phys. Commun. 191, 159 (2015).
[114] J. de Favereau, C. Delaere, P. Demin, A. Giammanco, V. Lemaître, A. Mertens, and M. Selvaggi (DELPHES 3 Collaboration), DELPHES3, A modular framework for fast simulation of a generic collider experiment, J. High Energy Phys. 02 (2014) 057.

[115] J. Alwall, M. Herquet, F. Maltoni, O. Mattelaer, and T. Stelzer, MADGRAPH5: Going beyond, J. High Energy Phys. 06 (2011) 128.

[116] A. Alloul, N. D. Christensen, C. Degrande, C. Duhr, and B. Fuks, FeynRules2.0-A complete toolbox for tree-level phenomenology, Comput. Phys. Commun. 185, 2250 (2014).

[117] E. Boos et al., Generic user process interface for event generators, arXiv:hep-ph/0109068.

[118] F. Maltoni, K. Paul, T. Stelzer, and S. Willenbrock, Color flow decomposition of QCD amplitudes, Phys. Rev. D 67, 014026 (2003).

[119] M. E. Peskin, How to turn a hep-ph paper into a simulation, West Coast LHC Theory Network, UC Irvine Meeting, 2007.

[120] J. H. Collins, K. Howe, and B. Nachman, Extending the search for new resonances with machine learning, Phys. Rev. D 99, 014038 (2019).

[121] B. G. Lillard, T. Plehn, A. Romero, and T. M. P. Tait, Multi-scale mining of kinematic distributions with wavelets, SciPost Phys. 8, 043 (2020).

[122] H. Beauchesne and Y. Kats, Searching for periodic signals in kinematic distributions using continuous wavelet transforms, Eur. Phys. J. C 80, 192 (2020).

[123] B. Nachman and D. Shih, Anomaly detection with density estimation, Phys. Rev. D 101, 075042 (2020).

[124] A. Andreassen, B. Nachman, and D. Shih, Simulation assisted likelihood-free anomaly detection, Phys. Rev. D 101, 095004 (2020). 